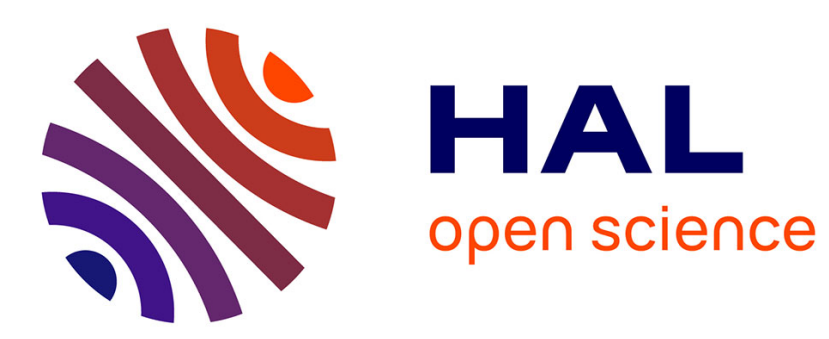

\title{
Les presbytériens et leurs élèves israélites en Perse: une brève rencontre (1871-1900)
}

\author{
Florence Hellot-Bellier
}

\section{To cite this version:}

Florence Hellot-Bellier. Les presbytériens et leurs élèves israélites en Perse: une brève rencontre (18711900). Jérôme Bocquet. L'enseignement français en Méditerranée. Les missionnaires et l'Alliance israélite universelle, Presses universitaires de Rennes, pp.324, 2010, Histoire. halshs-00672970

\section{HAL Id: halshs-00672970 \\ https://shs.hal.science/halshs-00672970}

Submitted on 22 Feb 2012

HAL is a multi-disciplinary open access archive for the deposit and dissemination of scientific research documents, whether they are published or not. The documents may come from teaching and research institutions in France or abroad, or from public or private research centers.
L'archive ouverte pluridisciplinaire HAL, est destinée au dépôt et à la diffusion de documents scientifiques de niveau recherche, publiés ou non, émanant des établissements d'enseignement et de recherche français ou étrangers, des laboratoires publics ou privés. 


\section{Les Presbytériens et leurs élèves israélites en Perse :}

\section{une brève rencontre (1871-1900)}

Poser la question de l'ancienneté de l'existence de communautés israélites en Perse/Iran ${ }^{1}$ semble paradoxal puisque la présence et l'histoire de ces communautés constituent d'importants chapitres de la Torah, de l'Ancien Testament et de l'histoire de l'empire perse achéménide. L'on sait en effet qu'après la déportation des Juifs de Samarie vers les villes mèdes à partir du milieu du vIII ${ }^{\mathrm{e}}$ siècle avant l'ère chrétienne, le temple de Jérusalem fut détruit par Nabuchodonosor en 586 et la population déportée à Babylone. En 538 le roi achéménide Cyrus autorisa les Juifs à retourner à Jérusalem ; un certain nombre d'entre eux choisirent de demeurer dans l'empire perse. Les juifs de Perse gardent aujourd'hui encore en mémoire les noms de la reine Esther et de son tuteur et cousin Mardochée, comme celui de Daniel dont le tombeau se trouverait à Suse et, au XIX ${ }^{\mathrm{e}}$ siècle, la lecture du livre d'Esther (le dernier des Cinq Rouleaux) était pratique courante dans les écoles des synagogues iraniennes. Après la conquête de la Perse par les armées musulmanes - en 642 de l'ère chrétienne - et l'imposition de l'islam aux populations de l'ancien empire sassanide (226-651), les juifs de Perse partagèrent avec les zoroastriens et les chrétiens le statut de dhimmis. Leur histoire devint alors celle de groupes minoritaires, intégrés dans les empires qui se succédèrent en Perse, mais tributaires d'une protection parfois aléatoire et des aléas politiques susceptibles de réveiller des intolérances masquées en temps ordinaire. Ainsi la situation des juifs fut-elle relativement bonne sous l'Ilkhanide Hulagü (1256-1265) mais s'aggrava sous Tamerlan (1370-1405). Sous les Safavides (1501-1736) qui imposèrent à la Perse le chiisme comme religion officielle, alternèrent périodes de persécution et de tranquillité. Les souverains qadjars (1779-1924) plus portés à la tolérance se révélèrent cependant incapables de la faire respecter et il se trouva souvent un mollah ou un modjtahed ${ }^{2}$ formaliste pour exploiter les situations de crises et les faiblesses du souverain, dénoncer chez les juifs «impureté et sacrifice d'enfants » et creuser le fossé de la discrimination sociale; il en fut ainsi lors des famines ou des épidémies de choléra particulièrement sévères au cours du dernier quart du $\mathrm{XIX}^{\mathrm{e}}$ siècle. Les souverains qadjars ne cessèrent pourtant pas de faire appel aux médecins juifs de Perse, et les grandes familles iraniennes ne conçurent pas leurs fêtes sans musique, exécutée le plus souvent par des musiciens juifs ${ }^{3}$; enfin on ne peut occulter dans la littérature iranienne l'influence et la beauté des œuvres des poètes écrites en judéo-persan.

Si l'on estime à $45000 / 50000$ individus le nombre de croyants se réclamant du judaïsme en Perse au tournant des $\mathrm{XIX}^{\mathrm{e}}$ et $\mathrm{XX}^{\mathrm{e}}$ siècles, ils représenteraient $0,5 \%$ de la population totale de Perse alors estimée à 10000000 d'individus. Dans son livre Between Foreigners and Shi 'is. Nineteenth-Century Iran and its Jewish Minority, écrit à l'aide d'ouvrages d'historiens juifs, des nombreux rapports conservés dans les archives de l'Anglo Jewish Association (AJA), de l'Alliance Israélite Universelle (AIU) ou des ministères français et anglais des Affaires étrangères, Daniel Tsadik a montré combien le judaïsme avait été tantôt toléré, tantôt rejeté dans la Perse du XIX $\mathrm{e}^{\mathrm{e}}$ siècle. Il faudrait y ajouter d'une part les archives des missionnaires presbytériens du Presbyterian Board for Commissioners for Foreign Missions (PBCFM) qui contiennent les lettres des presbytériens engagés en Perse au XIX ${ }^{\mathrm{e}}$ siècle dans le «Jewish work », d'autre part les lettres des missionnaires de la Church Missionary Society londonienne qui ont œuvré à Ispahan et à Chiraz où se trouvaient d'importantes communautés juives. On retiendra ici les écrits des presbytériens qui ont élargi la «Mission

\footnotetext{
${ }^{1}$ Reza Shah Pahlavi a imposé en mars 1935 la subsititution du terme « Iran » à celui de « Perse ».

${ }^{2}$ Dans le chiisme duodécimain, le modjtahed interprète le droit en attendant le retour du douzième imam.

${ }^{3}$ CHAOULl, A., Les musiciens juifs en Iran aux XIX ${ }^{e}$ et XX $X^{e}$ siècles, Paris, L'Harmattan, 2006.
} 
aux Nestoriens de Perse » reçue en 1871 des mains des méthodistes de l'American Board of Commissioners for Foreign Missions (ABCFM) en « Mission auprès des Arméniens, des Juifs et des Musulmans de Perse ». Leur expérience auprès des enfants juifs fut brève - à peine plus d'une trentaine d'années - mais féconde, entre 1871 et le tournant des $\mathrm{XIX}^{\mathrm{e}}$ et $\mathrm{XX}^{\mathrm{e}}$ siècles, moment où ils furent supplantés par l'Alliance Israélite Universelle (AIU). Les presbytériens eurent le souci d'individualiser leur travail et s'ils ont, à l'instar des autres missionnaires, créé des écoles, ils ont assez rapidement eu l'intuition que les écoles primaires/primary schools destinées aux enfants arméniens ne pouvaient être les mêmes que les écoles primaires destinées aux enfants juifs, et qu'elles différaient de celles qu'ils projetaient d'ouvrir aux enfants musulmans. En s'appliquant à forger dans les écoles juives un «nouvel homme », ils aspirèrent à donner à leurs élèves les moyens de faire évoluer leur condition. Les familles juives des deux «stations » presbytériennes de Téhéran et de Hamadan, se virent offrir la possibilité d'envoyer leurs enfants dans les écoles primaires presbytériennes jusqu'en 1900, et dans les collèges ou high schools qui perdurèrent au-delà de 1900. Ouvrir une école presbytérienne au sein du quartier juif d'Ourmieh, en Azerbaïdjan, s'avéra plus difficile. Sans occulter les difficultés et l'insécurité dans laquelle vivaient les communautés juives de Téhéran, Hamadan et Ourmieh, les missionnaires presbytériens demeurèrent inlassablement tournés vers l'avenir ; leur action s'inscrivit dans le mouvement qui, depuis la seconde moitié $\mathrm{du} \mathrm{XIX}{ }^{\mathrm{e}}$ siècle, porta les élites iraniennes, toutes religions confondues, à introduire, construire ou adapter, la modernité en Perse. Mais, en tant qu'étrangers d'une part, ils se trouvèrent exposés aux réactions xénophobes qui suivirent les cessions par Nasser ed-Din Shah (18481896) de pans de l'économie iranienne aux étrangers - concession Reuter en 1872 et concession des Tabacs en 1890 - ; d'autre part, dans leur ardeur à faire passer les juifs du judaïsme au christianisme, ils s'attirèrent les foudres des rabbins. Les écoles destinées aux enfants juifs en subirent le contre-coup.

Alors, quel bilan tirer de ces écoles presbytériennes, de caractère confessionnel marqué, qui formèrent pendant près de trente ans une minorité d'enfants juifs de Perse ? S'il fallait mesurer leurs résultats à l'aune de l'opposition des responsables des communautés juives, la réussite des presbytériens fut indéniable. Ont-elles ouvert la voie aux écoles de l'Alliance Israélite Universelle ? Ont-elles accéléré le passage au bahaïsme de certains de leurs élèves, passage déjà amorcé dans bon nombre de familles avant l'arrivée des presbytériens en Perse ? Elles ont en tout cas eu suffisamment d'influence pour donner à leurs élèves les moyens de redéfinir leur identité au sein de la société iranienne où mûrissait la révolution constitutionnelle de 1906.

\section{Appréhension contrastée de la communauté juive de Perse au XIX ${ }^{\mathrm{e}}$ siècle}

\section{Evaluation incertaine du nombre des Juifs en Perse au XIX ${ }^{\mathrm{e}}$ siècle}

Les estimations oscillent entre les indications du missionnaire anglican Joseph Wolff (17951862), converti du judaïsme et venu en Perse au cours des années 1920, considérées comme peu fiables, et celles d'Albert Confino envoyé en 1900 en Perse par l'AIU, qui semblent surestimées. Si l'on en croit le témoignage de J. L. Garland ${ }^{4}$ les juifs iraniens eux-mêmes sous-estimaient leur nombre pour des motifs variés, parmi lesquels la superstition tenait une grande place. Il faut donc croiser les estimations des voyageurs et témoins, en particulier des envoyés du Board of deputies of British Jews (JBD) fondé dès 1760, de la Church Missionary Society (CMS) fondée en 1799, de la London Society for Promoting Christianity among the Jews créée en 1824, de l'Alliance Israélite Universelle (AIU) fondée en 1860, de l'Anglo

\footnotetext{
${ }^{4}$ Garland, J. L., The Jews of Persia : their past and present condition, London, 1909.
} 
Jewish Association (AJA) fondée en 1871 et des presbytériens qui intervinrent en Perse au nom du presbyteran Board of Commissioners for Foreign Missions à partir de 1871.

En janvier 1880 le presbytérien J. H. Shedd reprit dans une lettre écrite d'Ourmieh le nombre de 50000 juifs en Perse «descendants des Dix Tribus », nombre déjà avancé par certains autres voyageurs. Dans son long rapport publié en 1896, un autre presbytérien, S. G. Wilson décompta 45000 juifs disséminés en Perse, en un arc de cercle englobant au nord et à l'ouest du pays, la province d'Azerbaïdjan - dont les centres urbains de Salmas, Ourmieh, Souldouz, Saoudjboulaq, Miandoab et Sakkez rassemblaient 1250 maisons, soit 6250 âmes - puis les villes de Senneh, Hamadan, Kermanchah, Kachan, Ispahan, Chiraz et Bouchehr auxquelles il fallait ajouter la province du Khorassan au nord-est du pays. La situation des juifs de Machhad, dans le Khorassan, était particulière puisqu'à la suite d'une accusation de blasphème portée contre leur communauté en 1839, lors d'un défilé religieux du mois de Moharram ${ }^{5}$, mois de deuil chiite, ceux-ci avaient été obligés de s'enfuir à Herat, ou de se convertir à l'islam. Il n'y avait donc officiellement plus de juifs à Machhad, bien que certains fussent revenus de Herat en 1857 lors de la guerre qui opposa Iraniens et Britanniques et que les presbytériens qui s'aventurèrent à Machhad eurent le sentiment que leur conversion n'était que superficielle.

La pétition transmise en 1873 au conseil de l'Anglo-Jewish Association à Londres par Baschi ben Simon, Ismail ben Suleiman et Fathollah ben Elia qui se disaient membres de la Commission des Sept (Représentants) de Téhéran ${ }^{6}$ comportait un tableau du nombre estimé de juifs et de synagogues dans chaque centre urbain :

\begin{tabular}{|l|l|l|l|l|l|}
\hline Noms de lieux & Familles & Synagogues & Noms de lieux & Familles & Synagogues \\
\hline Baneh & 30 & & Kerman & 20 & \\
\hline Borazjan & 20 & & Khoramabad & 30 & \\
\hline Borujerd & 40 & & Khunsar & 40 & \\
\hline Bushehr & 70 & 3 & Kurdistan & 250 & \\
\hline Djakouta/Chakudar & 25 & & Lar & 60 & \\
\hline Damavend & 30 & 3 & Mazanderan & 30 & \\
\hline Derab & 25 & & Miyandoab & 20 & \\
\hline Diyar & 25 & & Naghadeh & 40 & \\
\hline Djehané & 10 & & Nehavend & 30 & 2 \\
\hline Djein & 20 & & Nowbendegan & 20 & \\
\hline Dorudgah & 20 & & Oormiah & 30 & 4 \\
\hline Ferghana & 30 & & Qazvin & 40 & 2 \\
\hline Fazar/Fasa & 20 & & Salmas & 100 & \\
\hline Firuzabad & 50 & & Saqez & 60 & \\
\hline Garus & 60 & & Saoudjbulaq & 80 & \\
\hline Gilan & 30 & & Shiraz & 300 & 5 \\
\hline Gilyard & 30 & & Solduz & 10 & \\
\hline Golpaygan & 100 & & Teheran & 300 & 10 \\
\hline Hamadan & 450 & 4 & Tuyserkan & 30 & \\
\hline Hassanlu & 10 & & Yazd & 100 & 10 \\
\hline Isfahan & 400 & 10 & Zenjan & 50 & \\
\hline Jahram & 60 & & Ziendjioub & 30 & \\
\hline Kangover & 20 & & Zireh & 30 & \\
\hline Kashan & 150 & 6 & Zirtaré & 15 & \\
\hline Kerend & 20 & & Zohab & 20 & \\
\hline & & & & & \\
\hline
\end{tabular}

\footnotetext{
${ }^{5}$ Sur cet événement voir les détails rassemblés par TSADIK D., Between Foreigners and Shiis. NineteenthCentury Iran and its Jewish minority, p. 34.

${ }^{6}$ NETZER, A., « Persian Jews in the year $1875 »$ (en anglais) / «Esnad-e andjoman-e yehudian-e engelestan dar London » (en persan), Padyavand, t. 3, p. 83-91/ p. 255-260.
} 
Hamadan, Ispahan, Chiraz et Téhéran étaient les villes où la population israélite était la plus nombreuse. A propos de Téhéran, plusieurs rapports écrits au XIX ${ }^{\mathrm{e}}$ sièce laissent entendre que la population juive y était moins nombreuse qu'au XVIII ${ }^{\mathrm{e}}$ siècle. Elle était concentrée dans le quartier de 'Audjan/'Audjalan, à l'est de la ville. En 1872 le missionnaire James Bassett y compta environ trois cents maisons, dix synagogues - dont neuf situées dans de petites maisons - où n'entraient pas les femmes. Trois écoles étaient liées à ces synagogues, on y lisait les Écritures, en hébreu. J. Bassett découvrit aussi à l'extérieur de la ville, à Avhoch, une trentaine de familles ${ }^{7}$. Rassemblant plusieurs témoignages, Amnon Netzer décrivit en $1976^{8}$ le quartier juif de Téhéran au XIX ${ }^{\mathrm{e}}$ siècle, étroit et sale; les maisons avaient des murs de boue, des portes basses et des cours intérieures. La synagogue Ezra Yaqoub Cohen était la principale synagogue.

Le rapport adressé en 1883 au président de l'AIU par les juifs de Téhéran et transmis au ministère français des Affaires étrangères ${ }^{9}$ par le «Comité Central » donne, indirectement, bien des indications sur cette communauté :

«Un grand malheur a frappé la communauté juive de Téhéran.

Le 3 mars le juif Isaac Davisch a été trouvé en possession d'un objet de joaillerie qui, à ce que l'on prétendait, faisait partie des bijoux volés à Mirza Ali Khan fils de feu le grand vizir du Shah. Isaac Davish fut immédiatement incarcéré et mis à la torture du feu. Il déclara alors qu'il avait reçu le bijou du juif Haïm Isaac et d'une autre personne qui l'aurait volé. Haïm Isaac fut également arrêté mais quoique soumis à la torture il protesta de son innocence. L'autorité fit alors mettre en prison Baschi, fils de Simon, qui est le chef de la Communauté juive de Téhéran et dont le fils nommé Khodadad se prépare en ce moment à Londres pour être admis à l'École normale Israélite Universelle, une école française en Perse. On voulut faire signer à Baschi un engagement de faire verser par les juifs une somme de 125000 francs qui, suivant Mirza Ali Khan, serait l'équivalent des objets qui lui ont été volés. Baschi resta huit jours en prison et il fut soumis à la torture mais il refusa énergiquement de signer la reconnaissance qu'on lui demandait. Alors 14 juifs notables de Téhéran, parmi lesquels le rabbin en chef Abraham Hadji Baba, et ceux-ci finirent par signer une reconnaissance de 125000 francs. En outre la police et d'autres officiers inférieurs avaient reçu des juifs environ 200 livres sterling. La consternation des juifs (qui sans doute n'ont pas la somme demandée et dont probablement la vie est aussi en danger) est sans bornes ; ils se sont réfugiés en partie dans les écuries du ministre qui, suivant la coutume du pays, sont un asile inviolable. D'autres, hommes et femmes se sont réunis autour d'un grand canon qui est placé devant le palais du chah et où, suivant l'usage, se présentent les pétitionnaires qui veulent attirer l'attention du monarque. Mais jusqu'à présent leurs appels à la justice du chah sont restés infructueux. Le gouvernement anglais a télégraphié à son ministre à Téhéran d'employer ses bons offices en faveur des juifs 'au nom de l'humanité' ».

Le voyageur Rabbi David D'Beth Hillel, qui passa en Perse en 1828, pour aller en Inde, et dont le récit a été édité par Walter J. Fischel ${ }^{10}$, s'efforça de décompter la population des communautés juives de Perse. Il estima le nombre de familles à 2 799, soit 15000 âmes, deux fois moins que J. A. Bruwer ${ }^{11}$ à Constantinople à la même époque :

Tableau de Rabbi David D'Beth Hillel

\begin{tabular}{|l|l|l|}
\hline City & Estimated Jewish Families & Synagogues \\
\hline Bana & 10 & 1 \\
\hline
\end{tabular}

\footnotetext{
${ }^{7}$ BASSETt, J., Persia : Eastern Mission, p. 67.

${ }^{8}$ NeTZER, A., The Jews of Persia and Alliance in the late $19^{\text {th }}$ century, some aspects.

${ }^{9}$ Archives du ministère français des Affaires étrangères, (AMFAE), A. D. P., Perse, Carton 3, Paris 27 avril 1883, Comité central $\mathrm{n}^{\circ} 8505$.

${ }^{10}$ D'Beth HiLlel, Unknown Jews in unknown lands. The travels of Rabbi David d'Beth Hillel (1824-1832), p. 94-109.

${ }^{11}$ BRUWER, J. A., A resident at Constantinople, p. 39.
} 


\begin{tabular}{|l|l|l|}
\hline Saqiz & 15 & 1 \\
\hline Saugh Boulagh & 25 & 1 \\
\hline Taskala & 10 & \\
\hline Urmiya & 200 & 3 \\
\hline Salmas & 100 & 1 \\
\hline Bashqala & 20 & 1 \\
\hline Miyandoab & 15 & 1 \\
\hline Garus & 25 & 1 \\
\hline Senna & 300 & 2 \\
\hline Kaslan & 5 & \\
\hline Zohab & 40 & 1 \\
\hline Tuiserkan & 20 & \\
\hline Nahavend & 20 & 3 \\
\hline Kermanshah & 300 & 2 \\
\hline Hamadan & 200 & \\
\hline Téhéran & 100 & \\
\hline Isfahan & 300 & \\
\hline Kashan & 100 & 3 \\
\hline Shiraz & 700 & 1 \\
\hline Bushire & 200 & 1 \\
\hline $\begin{array}{l}\text { Persian Gulf } \\
\text { (Muscat) }\end{array}$ & 4 & \\
\hline $\begin{array}{l}\text { Persian Gulf } \\
\text { Sohar }\end{array}$ & 20 & \\
\hline
\end{tabular}

Les communautés juives de la province d'Azerbaïdjan firent l'objet de nombreuses descriptions.

Le voyageur Rabbi David d'Beth Hillel dépeignit la ville d'Ourmieh enserrée de murs bâtis sur une base de pierres, avec des rues étroites et de nombreux caravansérails ; on y travaillait le coton et la soie et les murs intérieurs de certaines maisons étaient joliment décorés. Deux cents familles juives habitaient un quartier sur une petite colline, autour de trois synagogues. Et le voyageur de remarquer que ces familles étaient assez riches, à l'instar de leur chef «Mulla Raphael, homme riche et honorable ». Cette réputation de richesse justifia plus tard les exigences financières de l'AIU à l'égard des juifs d'Ourmieh. A l'exception du missionnaire James Bassett qui avança en 1872 le chiffre de 2500 juifs à Ourmieh, la plupart des témoins estimèrent que le quartier de la colline des juifs d'Ourmieh rassemblait deux cent cinquante familles, soit un peu plus d'un millier d'âmes. En 1875 il y aurait eu quatre synagogues ${ }^{12}$.

De Salmas, Rabbi David avait retenu les larges rues, les «manufactures de coton de couleurs variées » et une centaine de familles juives groupées autour d'une jolie synagogue. En mars 1897, le presbytérien J. H. Shedd (1859-1895) estima le nombre des juifs de Kœnehchehr, non loin de Salmas, à cinq cents âmes.

Rabbi David décrivit aussi Soudjboulaq, au sud du lac d'Ourmieh, comme une large ville entourée de murs, où se trouvaient «quelques très jolies maisons, de nombreux caravansérails » et dont les habitants se répartissaient à peu près ainsi : 15000 musulmans, des arméniens et « vingt-cinq familles de riches Israélites autour d'une jolie synagogue ». En

\footnotetext{
${ }^{12}$ NETZER, A., Padyavand, t. 3, p. 88. Lettre addressée au Dr Allatini à Salonique. On admet cinq personnes par foyer.
} 
1895 le missionnaire presbytérien Benjamin Labaree ${ }^{13}$ s'arrêta à Soudjbulaq où il assista à une célébration dans la synagogue :

«At Soujbulak I attended morning prayers at the synagogue. On the door post, inclosed in a glass tube, were the Ten Commandments. In the one corner were the benches for circumcision and the bier. Each worshiper had bound on his arms and forehead with long leather cords, portions of the law, and thrown over his turban a thick white veil which hung down over his shoulders. The service, led by 3 or 4 rabbis, consisted in prayers from the Psalms and Talmud and reading of the law. Each man held an Old Testament in his hands, and they read in concert or responsively with frequent hearty amens to the prayers. The attitude was varied, being sitting, standing or bowing prostrate. The climax of the service was reached in the procession of the law. The manuscript roll of the law inclosed in a cylindrical case covered with scarlet broadcloth, topped off with two silver pomegranates, with pendant silver bells, such as hung from the high priest's robe. It was moved in procession through the synagogue, each one devotedly kissing it; and the women who had previously stood aloof, true to their nature, came forward to engage in this ceremony. Then they sang psalms and the people joined in petitions for the coming of the Messiah ».

Poursuivant sa route, Rabbi David donna une vision misérabiliste de Baneh (10 pauvres Israélites et une petite synagogue) à l'inverse de Saqqez dont la base des murs était en pierre et où une quinzaine de familles juives étaient regoupées autour d'une petite synagogue, comme à Miyandoab. A Garrous, dans la montagne, 25 familles israélites vivaient au milieu de 3000 familles musulmanes.

Senneh, capitale de la province du Kurdistan iranien et longtemps résidence de la famille kurde Ardalan, tranchait par sa taille et son importance, avec 50000 familles musulmanes et 300 familles juives autour de deux synagogues. La pierre était aussi utilisée dans les constructions. En 1888 le couple de missionnaires presbytériens Mr. and Mrs. Hawkes ${ }^{14}$ qui s'était rendu à Senneh à partir d'Hamadan transmit des chiffres légèrement inférieurs :

«Senneh, about 25000 souls, with the appearance and habits of a large town. Situated on the West side of a little plain, watered by the Širvan river and surrounded on all sides by mountains. [...] We had much trouble in getting settled as we wished to find a house where be likely to meet Moslems, Catholics and Jews. This was impossible and we kept the house in the catholic part of the city. We remained seventeen days and met many of the principal people of the three nationalities. There are 250 houses of Jews: they would like to have a school, but we could not find three heads of families bold enough to stand up ».

Dans son récit, Rabbi David citait ensuite Qasla avec 5 familles israélites et 1000 familles musulmanes, puis la ville d'Hamadan. Au centre d'Hamadan se trouvait une haute tour gardée par des israélites, appartenant aux deux cents familles de la ville (nombre avancé par Rabbi David), lesquelles disposaient de deux grandes synagogues pour leur culte. On y trouvait déjà des médecins estimés et de riches marchands et orfèvres. Le missionnaire John H. Shedd ${ }^{15}$ venu en 1870 d'Ourmieh à Hamadan, par la route caravanière de Tabriz à Bagdad, y décompta trente-cinq maisons arméniennes et un millier de maisons juives ; il insista sur la nécessité d'y ouvrir une «station presbytérienne ». Il luisembla aussi que « la médecine était aux mains des juifs ». Joseph Somekh envoyé en 1895 par l'AIU de Bagdad à Hamadan estima le nombre des israélites à 3000 âmes, qui se consacraient au commerce, à l'orfèvrerie et à la médecine ${ }^{16}$. La population totale d'Hamadan n'était pas loin d'atteindre 40000 individus.

\footnotetext{
${ }^{13}$ Archives Presbytériennes, (AP), vol. 18, Evangelistic Report, octobre 1895

${ }^{14}$ AP, Hamadan, June $22^{\text {nd }} 1888$.

${ }^{15}$ AP, Urumia May $25^{\text {th }} 1870$, J. H. Shedd to ABCM Boston.

${ }^{16}$ AIU, A1 B11, Hamadan 15 mars 1895.
} 
Les archives de l'AIU renferment une autre description de Senneh, en même temps que celle de Kermanchah ${ }^{17}$ :

«Seneh a 2500 israélites et Kermanchah, 1 800. Seneh compte environ 600 propriétaires de maisons et Kermanchah de 300 à 400 ; ils sont commerçants, vendent des étoffes grossières et de la mercerie contre du blé, des fruits, de la laine et des tapis. Ils occupent un quartier spécial. Dans le bazar leurs magasins sont mêlés à ceux des musulmans; ils fument le kalian ensemble à Seneh, vendent des fruits aux musulmans, se lavent dans le même bain et fréquentent le même coiffeur ».

D'autres voyageurs traversèrent Nehavend où se trouvait une vingtaine de familles juives, pour arriver à Kermanchah aux rues étroites, mais munie de deux ou trois belles places et de caravansérails. Trois cents pauvres familles israélites y subsistaient au milieu de 80000 familles musulmanes. Le missionnaire W. L. Whipple envoyé en Perse par l'American Bible Society au cours des années 1880 passa à Kermanchah en 1900. Il lui sembla que sur les 50000 habitants de la ville, 1500 seulement se réclamaient du judaïsme et 50, de l'Eglise chaldéenne, et qu'ils produisaient vin et arak. Trois ans plus tard le représentant de l'AIU se rendit à son tour Kermanchah ${ }^{18}:$ «Kermanchah est un rendez-vous de caravanes. Le quartier israélite de 135 maisons qui comptent au plus 1406 âmes, deux rues et quatre impasses, est séparé du reste de la ville par un ruisseau. On y voit des pierres tombales sans inscription. Audessus de la synagogue un local abrite Talmud et Torah. Les vieux rouleaux de la loi ont deux cent cinquante ans au plus ».

A Tuysserkan Rabbi David signala une vingtaine de familles juives, nombre un peu inférieur à celui de l'envoyé de l'AIU Joseph Somekh ${ }^{19}$ soixante-dix ans plus tard :

«A Tussurcane, ville de 4 à 5000 habitants chiites, construite non loin ancienne cité de Rudarvar où l'on trouve monnaies et pièces d'or, la communauté juive est d'environ 35 familles ou 200 âmes; au centre du cimetière est une tour octogonale de trois mètres de haut et dix mètres de périmètre, vue comme tombeau du prophète Habakuk ; c'est un lieu pèlerinage pour les musulmans et les juifs, car il guérirait les enfants. Le petit temple renferme dans la guéniza une dizaine de rouleaux de la Loi, les juifs ont dû arriver il y a plusieurs siècles. Autout de Tussurcane les israélites sont agents actifs de l'exportation de produits agricoles coton, blé, huile de ricin, tabac, mûriers, châtaigners, amandiers, meubles en bois et pipes.Une quinzaine de magasins israélites dans les bazars vendent de l'épicerie, mais il leur est interdit de vendre des tissus.Tussurcane appartient à le province de Malayer dont Dowlatabad est le chef-lieu ».

En 1903, à Boroudjerd, J. Somekh trouva « 800 âmes israélites, 120 familles habitant 80 maisons du quartier israélite, dont une vingtaine de familles (médecins et exportateurs) vivant dans l'aisance, 3 rabbins et un professeur d'hébreu. Les israélites de Boroudjerd sont très attachés aux traditions et très rigoureux dans leur observance. Les rabbins sont pauvres et ignorants ; le juif est considéré comme impur; il lui est interdit de chevaucher un cheval ${ }^{20} »$. La synagogue y avait été démolie en 1889, et le gouverneur Zell es-Soltan autorisa plus tard sa reconstruction.

Avant 1830 Rabbi David avait ajouté : «A Gavaré, Kerende, Kasr, 12, Zohab, Sarpol et Bouchehr se trouvent respectivement 18, 30, 12, 16, 14 et 200 familles israélites ». Il accordait à Chiraz 700 familles « soumises à une grande oppression », à Ispahan, 300 familles et à Kachan, 100 familles israélites.

\footnotetext{
${ }^{17}$ Dossier « Les juifs d'Iran », Les Cahiers de l'Alliance Israélite Universelle, n ${ }^{\circ}$ 8-9, septembre 1994.

${ }^{18}$ AIU, Hamadan 22 avril 1903, n 129.

${ }^{19}$ AIU, Hamadan 10 février $1903, \mathrm{n}^{\circ} 106$

${ }^{20}$ AIU, Hamadan 26 mai 1903, no 112.
} 
Un recensement effectué en 1882 et cité par A. Hountun-Schindler ${ }^{21}$, repris par Daniel Tsadik, donnait à Ispahan de 5306 à 6402 israélites, soit 7\% du total de la population de la ville. Le nombre de 6000 est le nombre le plus souvent repris pour Ispahan, comme celui de 5000 israélites pour Chiraz.

\section{Le judéo-persan et les écoles liées aux synagogues}

Chaque communauté juive devait avoir une école dans laquelle l'enseignement était prodigué en persan sur des textes écrits en hébreu. Si un assez grand nombre d'hommes pouvaient lire l'hébreu, seul un petit nombre de rabbins et de docteurs écrivaient l'hébreu et le judéo-persan, ainsi que l'atteste Arthur de Gobineau ${ }^{22}$, même s'il confond les langues puisque le syriaque parlé par les Chaldéens était une variante de l'araméen :

«La plus grande partie (des Juifs), à la vérité, s'occupe uniquement de soins matériels [...]. Mais on leur retrouve, en Asie comme ailleurs, cette énergie morale, cet orgueil religieux qui les élève et les fait surnager sur tant de catastrophes, et cela uni à une préoccupation vive, chez quelques-uns d'entre eux, de leurs dogmes, de leurs livres, de leurs sciences. [...] Les juifs ont des docteurs dont quelquesuns, en fait de connaissances talmudiques et philosophiques, sont très savants. J'ai été frappé d'un étonnement véritable le jour où l'un de ces érudits m'a parlé avec admiration de Spinoza et m'a demandé des éclaircissements sur la doctrine de Kant.[...] Pour des intérêts dogmatiques, pour des points doctrinaux, pour des questions de droit civil, ils se maintiennent en rapports constants avec le Grand Rabbin de Jérusalem qui décide souverainement de toutes les questions litigieuses. Très au courant des noms et de la façon de penser de leurs coreligionnaires européens les plus puissants, les juifs sont visités dans l'Inde et en Perse par des missionnaires ou plutôt des collecteurs qui recueillent parmi eux, pour les juifs de Jérusalem, des aumônes qui ne sont pas refusées. [...] Non seulement leurs correspondances traitent de questions d'intérêt ou de nationalité, mais elles ont aussi pour objet la discussion de points de doctrine et même l'échange de productions littéraires tantôt, mais rarement en hébreu proprement dit, tantôt en chaldéen, ou araméen et avec des recherches d'élégance linguistiques raffinées ».

Plusieurs voyageurs ou missionnaires témoignèrent au $\mathrm{XIX}^{\mathrm{e}}$ siècle d'une pratique ininterrompue de l'hébreu: le missionnaire Joseph Wolff fit remarquer que les rabbins d'Ourmieh parlaient un hébreu raffiné ; les missionnaires Stern et Sternschuuss entendirent parler hébreu lors de leur passage à Boroudjerd et Kazeroun en 1845 et 1846 ; deux ans plus tard le voyageur Israel ben Yoseph Benyamin pria en hébreu avec les juifs de Bouchehr, Yazd, Kachan et même à Mechhed ${ }^{23}$, comme Ephraïm Neumark en 1880 à Kermanchah, Nehavend, Boroudjerd, Tuyserkan, Damavend et Barforouch ; Joseph Lotka, converti et envoyé à Hamadan comme missionnaire au début des années 1880, y conversa en hébreu avec les juifs de la ville.

A Kachan, Hamadan, Ispahan, Chiraz et Kerman, les juifs parlaient le persan ou des dialectes apparentés au persan. Gilbert Lazard ${ }^{24}$ fait remarquer que le judéo-persan était la langue vernaculaire des Iraniens de religion juive ; elle avait rang de première ou de seconde langue ; ce judéo-persan - persan écrit à l'aide de l'alphabet hébreu - était la langue de la littérature israélite de Perse, qui faisait ainsi partie de la littérature persane. Des inscriptions, des exégèses de la Bible, des traductions de commentaires, des poèmes en persan classique comme ceux de Shahin à Chiraz au XIV siècle, les chroniques de Babay ben Lotf au XVII siècle ou de Babay ben Farhad au XVIII ${ }^{\mathrm{e}}$ siècle, ont été conservés ; ils appellent ainsi à nuancer

\footnotetext{
${ }^{21}$ Hountun-Schindler, A., Eastern Persian Irak, p. 119-120.

${ }^{22}$ GobineaU, A. de, Les religions et les philosophies dans l'Asie centrale, chapitre IV. A. de Gobineau fut à deux reprises en poste à Téhéran, de 1855 à 1857 et de 1862 à 1864.

${ }^{23}$ Le récit de son voyage fut publié en 1859.

${ }^{24}$ LAZARD, G., «The dialectology of Judeo-Persan » / « Gouyech chenassi farsihoud », NETZER, A., Padyavand, t. 1, p. 33-59 (en anglais) / p. 133-149 (en persan).
} 
les jugements trop hâtifs de certains voyageurs ou les impressions tirées de la lecture des suppliques adressées aux consuls étrangers, dépeignant les communautés israélites iraniennes comme illettrées et ignorantes.

Les écoles traditionnelles étaient au $\mathrm{XIX}^{\mathrm{e}}$ siècle surtout fréquentées par les garçons, à quelques exceptions près, à partir de l'âge de quatre ans jusqu'à la bar-mitzva. L'école pouvait être dans un bâtiment lié à la synagogue, ou se trouver dans la maison du rabbin. Les enfants étaient assis à même le sol. Les enseignants pour lesquels il n'existait pas de formation spécifique - quelques très rares enseignants se formaient à Bagdad - basaient leur enseignement sur les livres saints et les livres liturgiques, au centre desquels se trouvaient la Bible et les commentaires traduits en judéo-persan. Tous les enfants étaient initiés à la loi halakhah, certains cherchaient à connaître leur histoire, et le livre d'Esther était souvent sollicité ; il était surtout fait appel à la mémoire et à un apprentissage répétitif, très peu aux controverses et discussions intellectuelles.

\section{Le sarparast, intermédiaire entre les communautés non musulmanes et les autorités}

Les chefs de familles israélites n'étaient pas uniquement de pauvres gens. Comme l'indiquent les voyageurs, il y avait en effet des orfèvres qui travaillaient l'or ou l'argent, des bijoutiers, des médecins réputés à Hamadan et appelés à Téhéran auprès de la famille royale, des propriétaires de magasins dans le bazar d'Hamadan - mais pas ailleurs semble-t-il des vendeurs de soie à Ispahan et Téhéran. Ceux-là bénéficiaient de la considération des grandes familles iraniennes. Les revendeurs et colporteurs étaient plus discrédités, les producteurs d'alcool et de vin de Chiraz aussi. Les orchestres de musiciens juifs enchantaient les invités des grandes familles et les compositions musicales des israélites de Perse étaient d'une grande qualité ${ }^{25}$.

Mais il est vrai qu'il se trouvait souvent un musulman pour profiter des périodes de crise ou de faiblesse du gouvernement pour accuser les israélites de blasphème ou d'assassinat d'enfant, surtout pendant le mois de Moharram, mois de deuil sacré pour les chiites. Les juifs, ainsi accusés, n'avaient souvent d'autre solution que de se réfugier auprès d'un grand personnage ou de se convertir à l'islam. C'est ce qui s'était passé à Tabriz à la fin du XVIII siècle et à Machhad en 1839. Les consuls étrangers ou les associations de soutien aux israélites du monde telle que l'AJA et l'AIU, reçurent de communautés iraniennes de vibrants appels à l'aide ${ }^{26}$. Ces dernières crurent que le sarparast, dont la fonction fut instituée au cours de la décennie 1850, leur apporterait la protection et la sécurité auxquelles elles aspiraient et les protègerait des avanies qu'elles subissaient. Le sarparast, appelé souvent gouverneur des chrétiens, se démarquait du kargozar, agent du ministère des Affaires étrangères, en ce qu'il avait pour mission de s'interposer et de jouer le rôle de médiateur au nom du souverain qadjar, dans les conflits qui affectaient les minorités religieuses ou dans les conflits susceptibles d'opposer une minorité religieuse à la majorité chiite dominante. Le kargozar, quant à lui, gérait les situations conflictuelles dans lesquelles était impliqué un étranger. Il siégeait au tribunal consulaire quand un Iranien était impliqué avec un étranger dans une affaire donnée. De ces deux fonctions, la plus pesante pour les minorités juives ou chrétiennes fut rapidement celle du sarparast dans la mesure où elle représenta un maillon supplémentaire dans la hiérarchie des 'protecteurs' et une nouvelles source de taxes ou de cadeaux à offrir pour s'attirer la bienveillance du sarparast. Le sarparast dont la nomination à Téhéran donnait lieu à d'âpres marchandages, se trouvait aux côtés du gouverneur. La présence d'un sarparast ${ }^{27}$ est attestée à Hamadan, pour les juifs, comme à Ourmieh, pour les chrétiens, mais lors de l'arrivée d'un sarparast à Hamadan en 1881, le missionnaire J. W.

\footnotetext{
${ }^{25}$ ChaOulli, A., op. cit.

${ }^{26}$ Daniel Tsadik a rassemblé et commenté l'ensemble de ces appels dans son ouvrage publié en 2007.

${ }^{27}$ TSADIK, D., op. cit., p. 50-51.
} 
Hawkes ${ }^{28}$ signala que les juifs décidèrent de l'ignorer et de s'adresser directement au gouverneur. Il ne semble pas que le sarparast d'Ourmieh ait été aussi chargé des juifs d'Ourmieh. Il ne semble pas non plus qu'il y ait eu un sarparast partout où se trouvait un groupe religieux minoritaire. En tout cas la protection du sarparast dépendit de son degré d'honnêteté, et de son origine.

\section{La mission presbytérienne en Perse et les écoles ouvertes aux enfants juifs}

La mission presbytérienne et le judaïsme en Perse dans le dernier quart du XIX ${ }^{\mathrm{e}}$ siècle

Les missionnaires presbytériens américains ne furent pas les premiers missionnaires protestants à intervenir en Perse. Dans la mémoire collective sont gravés les noms de Henry Martyn et de Joseph Wolff, le premier parce qu'en revenant d'Inde il traduisit le Nouveau Testament en persan, le second, Joseph Wolff, israélite converti, parce qu'il vint en Perse à deux reprises, en 1824 et de 1828 à 1832, en manifestant une certaine extravagance. L'American Board of Commissioners for Foreign Missions (ABCFM), fondée en 1810, avait chargé en 1831 Eli Smith et H. G.O. Dwight missionnaires dans l'Empire ottoman d'effectuer un voyage d'investigation en Perse. Les deux hommes préconisèrent dans un premier temps de «revitaliser» ou de provoquer un renouveau dans l'Église d'Orient, dite Église nestorienne, dont le patriarche résidait au XIX ${ }^{\mathrm{e}}$ siècle à Qotchanès dans le Hakkari ottoman et dont quelque vingt-cinq mille fidèles résidaient en Azerbaïdjan iranien. Il serait ensuite possible d'évangéliser les musulmans. Le Rev. Justin Perkins fut envoyé à Tabriz où il arriva en 1834 en compagnie de son épouse et du missionnaire Haas de la Mission de Bâle. Le Dr. Asahel Grant mit son savoir-faire médical au service de la mission ; il arriva l'année suivante à Tabriz, avec son épouse. Les deux couples s'installèrent à Ourmieh la même année, au milieu des chrétiens 'nestoriens' auxquels ils proposèrent leur propre lecture de l'évangile. Quelques années après, en 1841, les lazaristes catholiques français s'installèrent aussi à Ourmieh pour revitaliser les six mille chrétiens chaldéens de la province. Les missionnaires méthodistes qui avaient fait venir une presse munie de caractères syriaques d'où sortirent un journal destiné aux populations de langue syriaque Les Rayons de lumière et des livres scolaires et liturgiques - rédigés en néo-syriauqe, langue accessible à tous, redoublèrent d'efforts. Se détournant de leur but premier, ils favorisèrent la fondation en 1855d'une Église protestante séparée, l'Evangelical Church, destinée à accueillir les transfuges de l'Église d'Orient, devenus protestants.

Parallèlement à ces efforts, la Church Missionary Society londonienne ou CMS, fondée en 1799, à envoyer des missionnaires en Perse ; en 1869 Robert Bruce répondit à l'appel et une mission de la $C M S$ fut fondée à Djolfa d'Ispahan en 1875. Le Dr. E. F. Hoernle le rejoignit en 1880, puis Miss Wilson en 1889, tandis que Mary Bird s'installa à Yazd au début du XX siècle. Leur objectif était clairement de s'adresser aux Arméniens.

Un certain nombre de communautés israélites avait reçu au cours des années 1840 la visite de missionnaires envoyés à partir de Bagdad, tels que H. A. Stern et P. H. Sternschuss. Leurs rapports soulignèrent l'insécurité dans laquelle vivaient les communautés juives de Perse, le discrédit attaché au métier de revendeur, l'estime dans laquelle les notables musulmans tenaient les médecins juifs et l'attrait du babisme sur les juifs de Chiraz et d'Hamadan.

En 1871 l'ABCFM transféra les activités de sa mission en Perse au Presbyterian Board of Commissioners for Foreign Missions (PBCFM). Les presbytériens élargirent leur champ d'action, en faisant de la primitive Mission aux Nestoriens une Mission aux Arméniens, Musulmans et Juifs de Perse. Comme le déclara en 1884 le missionnaire S. G. Wilson lors de

\footnotetext{
${ }^{28}$ AP, Hamadan December $6^{\text {th }} 1881$.
} 
la célébration à Ourmieh du Jubilé de la mission américaine en Perse ${ }^{29}$, le champ d'action de la mission se confondit avec la Perse tout entière, à l'exception du territoire confiée à la Church Missionary Society - Ispahan, Chiraz, Bouchehr. Les presbytériens ouvrirent de nouvelles stations, à Téhéran en 1872, à Tabriz en 1873, puis à Hamadan en 1882, sans abandonner la station d'Ourmieh ouverte depuis 1834 . Un certain nombre de missionnaires y furent affectés, aidés par des indigènes ou natives, bien souvent convertis. D'autres indigènes résidaient dans les sous-stations que les missionnaires visitaient lors de tournées annuelles (touring work). Au début des années 1880 la mission se scinda en une East Persia Mission et une West Persia Mission, qui évoluèrent avec une certaine indépendance l'une de l'autre. Elles se déployaient de part et l'autre d'une ligne imaginaire tracée de Senneh/Zendjan à Anzeli sur la mer Caspienne. L'étendue du champ d'action musulman justifia le nombre important de missionnaires affectés aux deux missions de Perse ainsi qu'une organisation très rigoureuse du travail missionnaire autour de trois piliers : évangélisation, éducation et travail médical, auquel vint s'ajouter celui de l'itinération (touring). Ces grands domaines d'activités se subdivisèrent eux-mêmes en «nestorian work, armenian work, jewish work ou moslem work ». Ce n'est donc qu'à partir de 1871 que les presbytériens envisagèrent de scolariser les enfants juifs de Perse et d'évangéliser leurs communautés.

\section{Création d'écoles presbytériennes au sein des communautés juives de Perse}

Dans le long rapport écrit à l'issue de sa visite aux missions presbytériennes de Perse en 1894, Robert E. Speer, secrétaire du PBCFM, tenta une synthèse des avis que lui avait confiés chaque missionnaire engagé dans une tâche d'enseignement (educational work). Diffuser le savoir et la civilisation, ou former des personnalités susceptibles d'assister les missionnaires dans leur tâche, venaient au second plan. J. H. Shedd à Ourmieh avoua que son but était « to raise up Christian leaders, men and women who will mold and guide their age, rise above circumstances and create for themselves a Christian environment». A Téhéran le missionnaire Ward s'était fixé deux objectifs : "To get and keep boys under Christian influence and to train preachers and teachers ». A Hamadan Mr. Watson s'en était fixé trois : «The conversion of the pupils, training good servants for servants of Christ, preparing generally for the coming of transition of Persia into a new life of liberty and enlightenment". Et Robert Speer de conclure que si la priorité des missionnaires était de transmettre l'Évangile au monde et de mettre sur pied, partout dans le monde, une Église locale, leur tâche éducative consistait à évangéliser les élèves non chrétiens et à faire émerger des communautés des leaders chrétiens. Et pour se faire, les presbytériens privilégiaient les unités de petite taille au sein desquelles les missionnaires enseignants pourraient marquer les élèves de leur empreinte $^{30}$.

L'ouverture d'écoles dans les quartiers juifs de Téhéran et Hamadan par les presbytériens répondit parfaitement à ces ambitions. A Ourmieh il en alla un peu différemment.

A Téhéran, en 1872, le missionnaire James Bassett aurait répondu favorablement à une requête de femmes israélites et accueilli quelques-uns de leurs enfants dans l'école presbytérienne, ouverte depuis 1873 aux enfants arméniens, près de la porte de Qazvin, à un demi-mile du quartier juif. En 1875 une nouvelle école fut ouverte dans le quartier Lalezar ; elle accueillit neuf élèves pensionnaires. Quatre ans plus tard, les presbytériens transférèrent ailleurs l'école arménienne. Ils louèrent une maison dans le quartier juif pour en faire une école qui puisse être largement fréquentée, en fin d'après-midi, par les enfants juifs de Téhéran. Fermée par manque de fonds en 1880, celle-ci fut réouverte en 1881, sous la direction de Mirza Baba et d'un converti nommé Nouroullah, fils de médecins juifs réputés,

\footnotetext{
${ }^{29}$ AP, Rapport lu lors de la célébration du cinquantenaire de la mission en Perse à Ourmieh en novembre 1884.

${ }^{30}$ SPEER, R. E., Report on the Persia Missions of the Presbyterian Board of Foreign Missions, p. 38-43.
} 
dont le patronage permit à l'école de surmonter les difficultés suscitées par certains rabbins. Au début de l'été 1882, l'école fut confiée à un converti arménien - un certain Michail aidé d'un autre arménien, Caspar ${ }^{31}$ - qui préparèrent la rentrée de septembre 1882 . De son côté Mrs. Basset, femme du persbytérien James Bassett, prit l'initiative de proposer des rencontres régulières aux petites filles du quartier juif et à leurs mères, dans la maison de Mirza Baba. En novembre 1883, sur les vingt-quatre femmes qui assistaient à ces réunions, huit demandèrent à apprendre à lire.

Ces initiatives perdurèrent, même si Nouroullah partit à Londres où il intégra la Hebrew Missionary Training Institution, et les rapports des missionnaires pour 1885 et $1886^{32}$ annoncèrent cinquante-et-un élèves dans l'école ouverte aux garçons : le dénommé Caspar avait été affecté à la boarding school, Baba, Mordechaï et Shuser Ullah à la Jewish Boys' school. Ces écoles durent porter leurs fruits si l'on en croit les menaces dont elles furent l'objet. En 1890, les presbytériens mirent à profit une certaine accalmie pour ouvrir une école primaire dans le quartier juif de Téhéran ${ }^{33}$; elle était bien vivante encore en 1893 :

«On oct. $8^{\text {th }} 1890$ a primary school was opened in the Jewish quarter of Teheran which has suffered no persecution and has perhaps succeeded better than could have been expected, having an attendance at present of 25 or 30 . On the evening of the same day the Jewish night school for young men was reopened without persecution. From the time the Jewish schools were opened till the summer vacation, a Sunday afternoon service was kept up in the school room, attended for the most part by the pupils but to some extent by others also. Some of the ladies of the mission, in so far as they could, attended this service, taking a separate room talking and reading to the Jewish women ».

Les réunions hebdomadaires de la Sabbath School rassemblaient par ailleurs des jeunes garçons «Jews, Parsees, Chaldeans and Armenians » qui chantaient des hymnes en persan, et défiaient la chaleur estivale en se retrouvant le matin, à 7 heures ${ }^{34}$. Les jeunes filles étaient accueillies dans une pièce séparée. Il y avait donc incontestablement une forte émulation. Deux nouveaux enseignants d'origine israélite furent affectés à l'école de garçons du quartier juif : Mirza Arestou dont les presbytériens apprécièrent la fidèlité et Mirza Khodadad qui travailla un moment comme interprète dans l'affaire de la Régie des Tabacs ${ }^{35}$.

Dans les statistiques données par le presbytérien L. E. Esselstyn pour 1891, figurèrent les enfants juifs qui fréquentaient les écoles presbytériennes de Téhéran ; le nombre d'élèves juifs apparaît inférieur à celui des enfants arméniens, mais supérieur à celui des enfants musulmans enseignés par les presbytériens, (les élèves «fire hippers» sont sans doute les enfants zoroastriens) :

Statistics Teheran boys'schools 1891 :

\begin{tabular}{|l|l|l|l|}
\hline Statistics & $1889-1890$ & $1890-1891$ & This fall \\
\hline Boarders & 51 & 36 & 41 \\
\hline $\begin{array}{l}\text { Day } \\
\text { scholars }\end{array}$ & 54 & 61 & 53 \\
\hline Armenians & & 68 & 52 \\
\hline Jews & & 13 & 17 \\
\hline Fire & & 4 & 4 \\
\hline
\end{tabular}

\footnotetext{
${ }^{31}$ AP, Téhéran 30 juin 1882 , lettre 261.

${ }^{32}$ AP, Téhéran 17 février 1887 : les salaires étaient de 15 tomans/mois pour Baba, 4 tomans 1/2/mois pour Mordechaï et 2 tomans/mois pour Shuser Ullah.

${ }^{33}$ AP, L. Esselstyn, Teheran. Evangelistic Work oct. $1^{\text {st }} 1890$-sept. $15^{\text {th }} 1891$.

${ }^{34}$ AP, Report of Teheran Sabbath School jan. $1^{\text {st }} 1890$ - oct. $15^{\text {th }} 1890$.

${ }^{35}$ Nasser ed-Din Shah dut annuler la Concession des Tabacs qu'il avait offerte aux étrangers, sous la pression des notables chiites qui se soulevèrent et interdirent aux Iraniens de faire usage de tabac.
} 


\begin{tabular}{|l|l|l|l|}
\hline hippers & & & \\
\hline Moslems & & 9 & 18 \\
\hline Americans & & 2 & 2 \\
\hline English & & 1 & 0 \\
\hline Nestorians & & 0 & 1 \\
\hline & & 87 & 94 \\
\hline
\end{tabular}

L'enseignement fut préparé ou soutenu par le travail mené par le Dr. Smith dans le dispensaire ouvert en 1891 dans le quartier juif ; tandis que le Dr. Smith y prodiguait des soins médicaux, d'autres missionnaires tentèrent de familiariser les patients avec le message évangélique : «Miss Dale has accompanied Dr. Smith and gathered the women and children for further teaching ». En 1892, l'école accueillit 23 'day scholars' et 12 'night scholars' enfants juifs.

Le 6 novembre 1893 Mrs. J. L. Potter put ouvrir une école primaire pour filles dans le quartier juif de Téhéran; cinq élèves furent d'abord inscrites, puis une trentaine, mais l'assitance demeura minime, en particulier à l'approche de la célébration de $\operatorname{Paswa}^{36}$ :

« A primary school for Jewish girls was started in their own quarter of the city this year. It has been a very interesting part of our work and one that has offered us considerable satisfaction. That is has succeeded so well is largely due to the determination and faithful character of the teacher. She is the daughter of one of the best families in the quarter, who had made the most of her educational advantages studying the first books in English with her brothers, who were pupils in the Boys' school of the Mission and having acquired a very creditable knowledge of the Persian language. One of her cousins who was baptized and received into the Christian Church in the early days of our mission here, having gone to England to be educated, was sent back to Persia by a British Society as a missionary to his own people. Through him Nusrat has heard the Gospel and she accepted Jesus as the messiah. [...] The ignorance of the girls surprised me, for I supposed Jewish children were carefully taught the Law and past history of their nation, according the express command given to Moses, but our teacher explained that girls and young women were excluded from the synagogue ».

Et Mrs. J. L. Potter de s'étonner que les jeunes élèves ne connussent pas Adam, le nom du premier homme mais aussi le nom signifiant «homme» en persan. Aussi l'enseignement porta-t-il d'abord sur l'histoire d'Abraham à Joseph et les élèves les plus éveillées furent-elles initiées à la suite de l'histoire jusqu'à l'époque de Salomon. Toutes s'exercèrent à mémoriser l'Évangile de Jean. L'élève la plus brillante, qui maîtrisa plusieurs alphabets, fut incitée à commenter les Écritures, ce qui permit à Mrs. Potter de l'amener à constater les erreurs des « héros de l'histoire juive, tel que Moïse ».

En 1895 Mrs J. L. Potter put se féliciter d'avoir appris les Dix Commandements aux jeunes filles. L. E. Esselstyn, quant à lui, dressa un bilan du travail realisé dans le quartier juif de Téhéran : «Jewish quarter Tehran: medical work of Dr. Smith, Jewish girls'school, boys' school of about 25 pupils, night's school of 25, without disturbance and a service has been held on Sundays a part of the year ».

L'American boys'school de Téhéran accueillit des garçons juifs : 13 sur les 70 élèves (29 musulmans et 28 arméniens), 13 encore en 1897 pour 70 musulmans, 50 arméniens et 1 zoroastrien, mais 2 seulement en 1898 pour 78 musulmans dont 7 princes iraniens.

La chute de 1898 fut donc brutale ! Dans son rapport du 29 décembre 1897 (qui couvrait la période 1896-1897) le missionnaire J. G. Wishard avait annoncé le transfert du travail auprès des juifs à d'autres missionnaires : "The 'London Society for propagation of the Gospel among the Jews' having entered upon its work in Tehran, it was deemed wise to transfer the

\footnotetext{
${ }^{36}$ AP, Mrs. J. L. Potter, Tehran Jewish girls'school 1893-1894.
} 
'Jewish Day and night school' to the care of the missionary of that society; it was done last winter ». Même allusion dans le rapport de Miss Annie Gray Dale qui écrivit en $1898:$ « 100 Jewish girls in the Jewish Quarter School (headed by Miss Campbell). This flourishing school has been handed over to an English society for Jewish work whose two missionaries are laboring in Tehran among the Jews ». L'ouverture d'une classe destinée à former des médecins procura aux presbytériens un peu de baume sur le cœur : «A new medical class was started last fall, consisting of 3 Jewish young men, all professing to be christian. Instruction gives them in return they help in hospital. Last returned to Hamadan ${ }^{37}$.

Joseph Cazès arriva à Téhéran en avril 1898 pour y ouvrir une école au nom de l'AIU ; elle accueillit trois cents élèves.

L'ouverture d'écoles de la mission presbytérienne à Hamadan fut plus aléatoire qu'à Téhéran. J. H. Shedd en avait souligné l'intérêt dès 1870 ; James Bassett en fit la demande au Presbyterian Board en 1877, puis de nouveau en 1878 ; en attendant, le Board of Foreign Bible Society envoya à Hamadan un millier de volumes religieux qui transitèrent par le Golfe persique. J. Bassett revint à Hamadan en octobre 1879 ; il promit aux récents convertis du judaïsme, dont Hezkiel Hyem et le Dr. Rahim « personnes intelligentes et influentes », ainsi que le Dr. Moosa et le Dr. Aga Jan l'ouverture d'une école dans le quartier juif. En 1880 Robert Bruce, de la Church Missionary Society londonienne, vint de Djolfa d'Ispahan à Hamadan ; les nouveaux chrétiens lui remirent une pétition qu'il envoya à la London Society for promoting Christianity among the Jews. Celle-ci décida l'envoi de deux missionnaires pour tenter d'implanter une mission à Hamadan. Les presbytériens en prirent quelque ombrage et, dans l'attente d'un financement de la station d'Hamadan par une communauté américaine, James Bassett envoya Mirza Ohana ouvrir un dépôt de livres de religion. A la fin de l'année 1881 le révérend Joseph Lotka, israélite converti de Lomberg, arriva à son tour à Hamadan pour trois ans ${ }^{38}$. L'année suivante les presbytériens purent enfin affecter $\mathrm{J}$. W. Hawkes (1880-1922) et son épouse à Hamadan ; ils firent leurs les réflexions du presbytérien J. H. Shedd ${ }^{39}$ : «In occupying new stations (Hamadan) in Persia, we have no right to expect great and wonderful results immediately. The Jews and the Moslems and all, in fact, will require long patience, but we shall reap if we faint not". J. W. Hawkes ouvrit immédiatement une école dans le quartier juif, laquelle fut fermée en janvier 1883, réouverte en février, refermée en 1884 et transferrée dans une maison louée par le pasteur Shimon, comme le notèrent Annie Montgomery et le couple E. W. Alexander après leur arrivée à Hamadan en 1883. L'école n'ayant jamais définitivement fermé ses portes, les enfants juifs étaient en 1885 capables de chanter des psaumes et de raconter l'histoire de Salomon. Mrs. Alexander entreprit à son tour d'offrir à l'ouest du quartier juif une école aux petites israélites d'Hamadan, laquelle fut tour à tour fermée, puis ouverte ${ }^{40}$ :

« The great majority of Jewish homes are ruled over by mothers who are ignorant and indifferent holding not to religion but to tradition and superstition with great tenacity. To visit them in their house - talk to them and try to interest them in the religion of Jesus is within this sphere of our work. To elevate these women will can only be done through Christian schools for their girls. My greatest hope centers in such a school for the Jewish girls ».

Treize petites filles fréquentèrenty l'école au cours de l'hiver 1888, vingt-sept en 1892 et quatre-vingt douze s'y inscrivirent en 1893. Elles s'initièrent à l'histoire tirée de la Bible

\footnotetext{
${ }^{37}$ Ces citations sont tirées des différents rapports écrits par les presbytériens de Téhéran à la fin de 1898 .

${ }^{38}$ WATERFIELD, R, Christians in Persia, p. 116.

${ }^{39}$ AP, J. H. Shedd, Ourmieh January $10^{\text {th }} 1882$.

${ }^{40}$ AP, Gertrude Alexander, Hamadan Report, 1887-1888.
} 
depuis Adam jusqu'à Joseph, à l'art du tricot et de la couture. Trois d'entre elles apprirent l'histoire du temple de Salomon. Mais les enseignantes déplorèrent l'âge précoce de leur mariage, 12 ans ou même moins, et certaines de leurs élèves fréquentèrent l'école avec un bébé dans les bras.

La High School ouverte en 1887 fut surtout fréquentée par de jeunes israélites et par quelques arméniens. Elle mêlait musulmans et juifs. En 1891, sur les quatre élèves juifs de la High School l'un se prépara «au ministère parmi son propre peuple ». La plupart des élèves se destinaient à la médecine.

Le programme de la Boys'school était assez varié ${ }^{41}$ : au premier niveau, à la lecture quotidienne de la Bible (chaque matin, pendant une demi-heure) s'ajoutaient l'étude de la Bible, de l'histoire, de la géographie, de l'arithmétique, de l'algèbre et de la grammaire anglaise. Au second niveau, tous les élèves étudiaient la Bible et l'arithmétique, mais se séparaient pour étudier la grammaire en arménien, en hébreu ou en persan. Au troisième niveau (senior) les presbytériens enseignaient la Vie du Christ, l'algèbre, la physiologie et la grammaire arabe. Mirza Hayem, l'un des quatre « native teachers », enseignait l'hébreu.

La Hamadan Sabbath school rassembla chaque semaine une quarantaine de garçons et de filles.

Le 24 octobre 1892 le presbytérien J. G. Watson écrivit un long rapport ${ }^{42}$ sur le Jewish work à Hamadan, dans lequel il s'en prenait à l'islam et à la condition féminine en Perse :

« In considering this general branch of Christs work in this city we observe three/two centers or lines of action. The Boys' school, the Jewish Girls' school and Saturday and Sunday services held in the Boys' School building. The first named is not untended for Jews alone but for boys of all classes, whether Jew, Moslem or Christian. It is given a distinct place and forms no part of my report. Next in order is the Jewish Girls'School located at the west end of the Jewish quarter. This is intended to be a center of influence for the improvement of Jewish home life. It is a much needed institution. From the comparatively advantageous position occupied by woman in Bible History we might expect to see and hear better of their state among the Jews in this year of our Lord 1892; but owing to the depressing atmosphere of Islamism which surrounds and presses close upon her here, or for whatever cause, her lot his little better than that of her sisters in Islam. This state and condition is to well known to need further mention. There is a need for such a school as we have, right in the head of the heart of the community which takes these daughters born to adversity in infancy and in the little while it has opportunity, surrounds them within sweet clearly influences of the Gospel, teaches them its truths and helps to open the windows and let the light which shall enable them to bear the burdens better and to look forward to something better for their children. Precarious tho its existence has been and disultary the attendance and vicissitudes many, still it exists to begin another year of work Oct. $17^{\text {th }}$ with a fair attendance. On the return of Rev. and Mrs. Hawkes to the field last fall, the latter was asked to take charge of the school, the native charge remaining the same as before in the hands of Rebecca Said and little Khanum. Mrs. Hawkes management has made a worked improvement in the appearance of the hair and in the orderly conduct of these daughters of Israel. During the year twenty seven were enrolled. They are instructed in the principal elementary branches, the Bible and catechism being chief text books. They are married so young that only small children are to be found in the school, tho a few has been gamered from faith Hubbard during these years and one at least has made public profession of faith in Christ. The difficulties are great. The little ones are frequently badly treated in going to and from school. If the Jewish community were only so compactly situated as the Armenian, this could not occur. As already stated the girls are married so young that they do not remain long enough in the school (with the exceptions noted) to do more than make a start. Apparently the only way to obviate these chief difficulties would be to have little schools in all compact parts of the community. The remaining line of work is the religious services, which are held in the Boys' school building adjoining

\footnotetext{
${ }^{41}$ AP, J. G. Watson, Hamadan october $21^{\text {st }} 1891$, Boys 'High School 1891.

${ }^{42}$ AP, J. G. Watson, Hamadan October 24th 1892, Report of Jewish work in Hamadan. Termes soulignés dans le texte par Watson.
} 
the residence of Mrs. Hawkes and located at the opposite end of the Jewish quarter of the Girs'school. A prayer meeting is held Saturday evening Sabbath school and Sabbath morning [...]. These services are attended mostly by those who are or were in the school and a few parents. During the two other midsummer months, when the school is closed and many away the attendance is greatly diminished ».

Il n'en demeurait pas moins que les missionnaires voyaient leurs efforts couronnés de succès quand un élève élevé dans le judaïsme faisait profession de foi chrétienne. J. G. Watson, comme d'autres missionnaires, le reconnut en 1893: "The two Hebrew boys of the class, brother and son of one of the most respectable physicians of Hamadan, have both confessed their faith and acceptance of Christ as their savior to us as individuals, tho they have not seen their way clear to accept public baptism because of the very strong opposition their father interposes ».

Les presbytériens d'Hamadan pensèrent le moment propice à la création d'une Église qui accueillerait les convertis du judaïsme, comme l'Evangelical Church accueillait depuis 1855 les convertis de l'Église d'Orient à Ourmieh, ou St Stephen Church les convertis de l'Église arménienne à Hamadan. En 1894 ils créèrent à Hamadan la Peniel Church qui démarra avec dix membres. En 1896 Annie Montgomery put se réjouir ${ }^{43}$ :

« The work in the Jewish quarter never seemed so promising as at present. Though the membership in Peniel church is not large, most of those enrolled are active, earnest workers. Mr Watson, the pastor, has managed among his numerous duties, to visit all the families in connection with the Church, and all the families in connection with the Boys' school in which there are 78 boys. He also arranged that by means of the members of the Church all the remaining families in the community should be visited, some of the members have completed their list and reported, but not all. The Sabbath School superintended by Dr. Holmes has a much larger attendance this year, as also the church service, which is held immediately after $»$.

Si les rapports écrits d'Hamadan en 1897 et 1898 permirent de constater une légère augmentation des effectifs scolarisés par les presbytériens, ceux de 1899 furent assombris par une sourde menace : celle de l'arrrivée à Hamadan d'une « Society of Old Jews from Paris ». L'AIU s'apprêtait en effet à ouvrir une école qui attira la moitié des effectifs des écoles presbytériennes de filles et de garçons ; seule demeura la Peniel Sunday School ouverte en 1894, qui rassembla encore vingt-cinq élèves et continua à être animée par Agha Hyem. Monsieur et Madame Bassan, de l'AIU, arrivèrent à Hamadan en 1900.

L'élan des écoles presbytériennes destinées aux jeunes israélites était cassé, à Hamadan comme à Téhéran.

A Ourmieh l'AIU n'ouvrit pas d'école dans le quartier juif. Le Rev. Whipple signala en janvier 1875 qu'un juif d'Ourmieh s'était converti au christianisme en Russie et qu'à son retour il avait entraîné derrière lui une quinzaine de familles du quartier juif. Les presbytériens leur affectèrent deux teachers. Miss K. Van Duzee arrivée à Ourmieh en 1885 ne cessa d'arpenter le quartier juif où elle constata que les maisons lui étaient ouvertes ; elle agit par touches successives, privilégiant la parole et la lecture de la Bible. Mais il n'y eut pas avant la révolution constitutionnaliste, annoncée à Tabriz dès 1905, d'écoles organisées comme celles de Téhéran ou d'Hamadan. Miss K. Van Duzee fit état de femmes chrétiennes qui allaient, comme elle, de maison en maison, telle cette Hannah qu'elle évoque en $1903^{44}$ :

"Our Bible woman Hannah is still devoting all her time to the Jews. She reports 27 young men, brides and girls reading in the Old Testament, one in the New Testament. Four have learned to read so well that they have graduated from her care. Six are learning to write. Into all their homes she goes daily

\footnotetext{
${ }^{43}$ AP, Annie Montgomery, Report Hamadan 1896.

${ }^{44}$ AP, Work for Moslem and Jewish women and girls, sept. 1902-sept.1903.
} 
and is continually holding Christ before them as the world's savior. We have noticed this year much more friendliness and cordiality on the part of Jews, than ever before and nearly always on Saturday different companies come to any room, requesting me to read them".

Le résultat fut suffisamment probant pour permettre à de jeunes israélites, garcons et filles, de rejoindre les classes ouvertes à Ourmieh par les presbytériens, dans des sections spéciales, à partir de 1905 et jusqu'à la Première Guerre mondiale.

Il est indéniable que les presbytériens formèrent dans leurs écoles, même brièvement, des élites israélites en Perse, à la fois par le programme d'études offert et par les méthodes employées. Les matières étudiées furent extrêmement variées, avec une approche d'un enseignement moderne scientifique, bien loin des «quelques notions de la Bible et d'une sorte de griffonnage de l'écriture hébraïque qui sont toutes les connaissances qu'on acquiert ici » dénoncés par Joseph Somekh en 1895.

L'étude des langues usitées en Perse ou de la langue américaine offrit de nouvelles perspectives aux élèves. L'étude de l'hébreu, étendu aux filles et aux garçons, conforta la communauté israélite de Perse dans son identité religieuse. Il n'en reste pas moins que l'ouverture d'un enseignement aux femmes et à leurs filles constitua sans doute le progrès le plus marquant, d'autant que les moyens d'atteindre les femmes furent multiples, depuis l'école jusqu'aux réunions informelles. Les femmes presbytériennes réussirent-elles à obtenir un recul de l'âge du mariage comme elles le souhaitaient? Ce n'est pas très sûr. Mais Dans les High Schools les enfants juifs apprirent à fréquenter les enfants arméniens, parfois même comme à Téhéran, les enfants musulmans. Dans la mesure où cette dernière expérience ne fut pas interrompue par l'arrivée des enseignants de l'AIU, elle fut favorisa la formation d'une communauté nationale en Perse.

La lettre adressée en 1903 par les juifs d'Ourmieh qui ne bénéficiaient pas d'écoles presbytériennes aux représentants de l'AIU, par l'intermédiaire de M. Cohen, prouva que, par défaut, l'enseignement des presbytériens était apprécié, en ce qu'il propageait la civilisation :

\section{«Monsieur le Président,}

De passage à Ourmieh, nos coreligionnaires de cette ville m'ont prié de m'unir à eux en faisant mes démarches auprès des intéressés en ce qui concerne la fondation dans leur ville d'une école sous le patronage et habile direction de la célèbre AIU et à quoi j'ai accepté de grand cœur.

Monsieurr le Président c'est la deuxième fois dans l'intervalle de trois ans que je passe quelques jours entre nos coreligionnaires de cette ville. J'ai pu constater de visu combien l'état intellectuel et moral de ces derniers laissait à désirer ; en comparaison des communautés sœurs chrétiennes, la communauté israélite se trouve bien inférieure tandis que les premières, grâce aux missions protestantes, catholiques et orthodoxes se relèvent par étapes dans l'échelle civilisatrice, la seconde s'enfonce dans l'ignorance et dans la misère et recule d'un pas de la civilisation. Les écoles, les talmuds, torahs même font défaut Mr le Président.[...]

Monsieur Lévy dans son rapport séparé vous a donné tous les détails relatifs, pourtant je crois bon de déclarer que sitôt que l'école sera fondée les communautés voisines de Salmas population israélite de 350 habitants, Sulduz [Souldouz] 800 habitants, Ichné [Ouchnou] 250, Saouchboulak [Soaudjboulaq] 600 habitants, fourniront de leur côté un nombre assez considérable d'enfants qui contribueront eux aussi au maintien de la susdite école.

Monsieur le Président, en dotant la communauté israélite du Kurdistan d'une école, vous aurez fait une œuvre humanitaire des plus méritées, vous aurez éclairé de la lumière civilisatrice de 7 à 8000 de nos coreligionnaires qui se trouvent dans une complète obscurité et combien ce millier d'enfants que vous aurez instruits ne vous bénira-t-il pas pour ce bienfait incalculable?

Dans l'espoir que notre prière, et celle de la communauté avec, sera reçue et agréée, je viens, Monsieur le Président, me soumettre à votre bienveillance ». 
La lettre comportait des propositions d'une contribution financière que l'AIU jugea insuffisante, car elle pensait que la communauté d'Ourmieh était parmi les plus aisées de Perse. L'AIU ne donna pas suite à la demande des juifs d'Ourmieh.

\section{Les reproches adressés aux écoles presbytériennes ouvertes aux enfants juifs}

Les premiers reproches provinrent de la communauté israélite elle-même.

En 1886 Mirza Baba, directeur de l'école ouverte dans le quartier juif de Téhéran, fut emprisonné sur ordre de Nasser ed-Din Shah après que ce dernier eut pris connaissance d'une que lui avait adressée le Grand rabbin de Jérusalem qui se plaignait de ce que les écoles presbytériennes détournaient les juifs de la foi de leur père. Le ministre américain, récemment arrivé à Téhéran (en 1883) intercéda et obtint la libération de Mirza Baba ${ }^{45}$.

Si les rabbins de Téhéran se plaignirent de l'installation d'une école presbytérienne dna le quartier juif, à Hamadan des femmes israélites s'inquiétèrent de ce que leurs filles risquaient de perdre leur foi dans les écoles presbytériennes ${ }^{46}$. En 1895 Joseph Somekh s'en prit aux contradictions internes des juifs de la ville d'Hamadan: «Orgueilleux, fanatiques et ignorants, ils deviennent blâmables par leur conduite et leurs mœurs si repoussants qu'elles donnent de l'horreur à tout étranger. Pendant qu'ils sont poursuivis et molestés par les musulmans, une lutte continuelle est levée parmi eux qui divise la partie rabbinique des autres. Les rabbins veulent les dominer tyranniquement; eux veulent secouer leur joug » ${ }^{47}$.

Cette inquiétude des rabbins fut justifiée dans la mesure où un certain nombre d'enfants des écoles presbytériennes adhérèrent au christianisme, ce qui était le souhait des presbytériens avant $1900{ }^{48}$. On retrouve ici le problème plus général posé par les conversions et les convertis en pays d'islam. En 1880, lors de la mort du Dr. Aga Jan, passé du judaïsme au christianisme, les «Old Jews » comme les appelèrent les presbytériens, refusèrent qu'il fût inhumé dans le cimetière juif d'Hamadan.

A Ourmieh où n'existait pas encore d'école presbytérienne pour les petites filles juives, les rabbins s'alarmèrent du porte à porte mené par des femmes attachées à la mission presbytérienne ou d'informels rassemblements tenus dans des maisons du quartier juif, comme en témoigna Miss K. Van Duzee ${ }^{49}$ :

«For Jewish women our faithful Bible woman Hannah has worked steadily. 16 or more pupils are learning to read and their daily lessons have occupied all her time except Saturdays which she devotes to general visiting. In the spring there was some opposition to her work and the leading men talked of it in the synagogues proposing to excommunicate all who allowed her to teach in their homes. She heard of this and told me afterwards that she wrestled with God in prayer that night and then nothing more has been heard of the opposition, while almost daily new ones are begging that she teaches their children to read ».

Mais de leur côté, les musulmans, inquiets ou jaloux de l'enseignement dont bénéficiaient ceux qui fréquentaient les écoles presbytériennes, les obligèrent à porter un morceau d'étoffe rouge sur leurs vêtements, à couper les boucles de leurs cheveux, à ne pas monter à cheval, à ne pas sortir les jours de pluie etc. La réaction la plus draconienne fut celle de Chaykh Mohammad Taqi Nadjafi (1846-1914) connu pour son hostilité à l'égard des juifs et des babis et pour sa participation à la campagne contre la Concession des Tabacs, qui édicta en 1889 à Ispahan une liste de treize articles discriminatoires destinés à isoler les juifs et à les empêcher

\footnotetext{
${ }^{45}$ AP, Teheran July $9^{\text {th }} 1886$, W. W. Torrence.

${ }^{46}$ AP, Mrs. Hawkes, Hamadan, Report 1892-1893.

47 AIU, A1 B11, Hamadan 15 mars 1891.

${ }^{48}$ A Ourmieh, W. A. Shedd se démarquera de son père J. H. Shedd sur ce sujet, au début du XX $X^{\mathrm{e}}$ siècle.

${ }^{49}$ AP, Report of woman's work in Ourmieh, September $1^{\text {st }} 1900-$ September $1^{\text {st }} 1901$.
} 
de se livrer au commerce ; le gouverneur Zell es-Soltan parvint, non sans difficulté, à les faire annuler.

Ignorant le sarparast qui ne leur évitait pas les persécutions, les juifs firent appel à Nasser eddin Shah et à ses ministres ; les presbytériens en firent autant, au nom de leurs convertis. C'est ainsi qu'ayant alerté les communautés israélites de Grande-Bretagne où Sir Moses Montefiore en relation avec Ronald Thomson à Téhéran se dit prêt à venir en Perse, ainsi que l'Alliance Israélite Universelle en France qui était alors présidée par Adolphe Crémieux, Sir Moses Montefiore et Adolphe Crémieux entreprirent des démarchesdes démarches auprès de Nasser ed-Din Shah pendant son passage à Londres et à Paris en 1873. Le 12 juillet Adolphe Crémieux lui remit la pétition ainsi rédigée ${ }^{50}$ :

«Voici qu'une triste et mémorable occasion va se présenter à nous. Dans plus d'un État, le rapport de notre secrétaire vient de nous l'apprendre, les persécutions religieuses existent et reparaissent ; mais les plus malheureux Israélites sont les Israélites qui vivent dans le royaume de Perse. En Perse où les Juifs, s'ils ne sont pas voués à la mort, comme le furent leurs aïeux au temps d'Assuerus, sont voués à la plus déplorable persécution, où on leur impose les travaux les plus vils, les plus odieux. Je ne puis pas vous tracer ce malheureux tableau; mais puisque par un événement qui semble miraculeux le monarque persan vient dans nos contrées, l'Alliance Israélite Universelle paraîtra devant lui, elle pourra l'aborder, elle pourra lui dire : « Nous venons vous demander de faire cesser la persécution qui, dans votre Empire, frappe les Israélites. Celui qui régnait sur la Perse il y a plus de dix mille ans avait signé, sans le savoir, la condamnation à mort de tous les sujets juifs qu'il avait alors ; celui qui règne aujourd'hui en Perse ne sait pas la situation déplorable dans laquelle se trouvent quarante mille Juifs, ses sujets. Au nom de ce Dieu qui vit toujours, du même Dieu qui protégeait les Juifs il y a vingt siècles, nous venons solliciter votre puissance royale, nous venons lui demander d'abolir l'odieuse, la déplorable dégradation dans laquelle végètent tant d'hommes dont les ancêtres furent protégés par un grand roi de perse et qui, dans l'état d'abjection où les tiennent d'abominables persécutions, inconnues de votre autorité, élèvent leurs voix suppliantes vers votre trône, comme vers le trône de Dieu.

Et dans une adresse écrite il lira l'incroyable récit, il verra l'affreuse peinture des maux que subit cette nombreuse population sans appui. L'appui de Dieu ne manquera pas à notre pieuse démarche et nos neveux pourront joindre à la délicieuse histoire d'Esther sauvant des Juifs de Perse une méghilla non moins touchante de l'Alliance sauvant au XIX ${ }^{\mathrm{e}}$ siècle les Juifs persans, sujets de Nasser ed-Din ».

Nasser ed-Din Shah s'engagea non seulement à protéger les juifs de Perse, mais aussi à faciliter l'ouverture d'écoles parmi eux. Il revint en Perse, mais aucune école ne vit le jour. Aidés des presbytériens qui s'appuyèrent sur les ministres anglais, français et américains à Téhéran, les israélites de Perse obtinrent, comme les arméniens ou les chaldéens, ce que certains appelèrent - à tort - la liberté religieuse, et qui fut plutôt la possibilité légale pour les non musulmans de passer d'une religion à l'autre. Cet agrément obtenu en 1880 à la suite de sévices subis par un israélite d'Hamadan, Yary Attar (ou Ahtar), après sa conversion au christianisme, fit l'objet d'un ordre du ministre Mostowfi el-Mamalek adressé au gouverneur d'Hamadan, en date du mois de djamadi el-awal 1297/janvier 1881. Il laissait les juifs devenir chrétiens si la communauté juive continuait à payer les taxes ${ }^{51}$.

En juillet 1889, lors de son troisième voyage en Europe, Nasser ed-Din Shah reçut à Londres une nouvelle délégation de juifs qui renouvelèrent leur demande d'écoles en Perse. Le souverain iranien s'y engagea verbalement à condition que ce fussent des écoles séculières. S'il oublia sa promesse, il tenta cependant de modifier la tradition qui permettait à la communauté musulmane de capter les héritages de ceux qui étaient passés du christianisme ou du judaïsme à l'islam, au détriment des familels des convertis.

\footnotetext{
${ }^{50}$ NETZER, A., The Jews of Persia and Alliance in the late 19th century, p. 14-15.

${ }^{51}$ AP, Teheran June 17th 1880, November 22nd 1880, J. L. Potter, Ourmieh February $10^{\text {th }} 1881$, B. Labaree.
} 
La requête qui lui avait été adressée à Londres faisait suite aux démarches accomplies par les diplomates étrangers en poste en Perse. Les presbytériens sollicitèrent successivement l'appui des diplomates anglais, puis américains après 1883, en cas de difficulté afférente au maintien de leurs écoles dans le quartier juif de Téhéran ou d'Hamadan. Ceux-ci se montrèrent très prudents; ils conseillèrent aux presbytériens de modérer l'ardeur de leur prosélytisme et même de bannir le prosélytisme de leurs écoles; conseillant un travail plus spirituel, ils n'intervinrent auprès du vazir ou auprès du chah qu'en cas d'injustices dument avérées (comme à Hamadan en 1875, 1892 ou 1901 ou à Téhéran en 1890).

Après l'ouverture définitive d'une légation à Téhéran en 1855 , les diplomates français ne reçurent pas d'instructions les invitant à protéger coûte que coûte les israélites de Perse, pas plus que les chrétiens d'ailleurs. Sur ce sujet les correspondances échangées sont très claires. Ponstuellement les ministres français en poste à Téhéran interrogèrent leurs supérieurs pour savoir quelle conduite adopter; les réponses de Paris furent, elles aussi, ponctuelles. Aussi fut-il surprenant de voir en 1879 le ministre français des Affaires étrangères conseiller à son représentant à Téhéran de soutenir les écoles de l'AIU, qui n'existaient pas en, à moins d'imaginer les démarches de l'AIU auprès du ministre, à la suite sans doute de demandes d'écoles en provenance des communautés israélites de Perse ${ }^{52}$ :

«Monsieur, le Comité central de l'Alliance Israélite universelle, dont le siège est à Paris, m'expose dans une lettre du 4 mars, les résultats de ses efforts pour répandre l'enseignement primaire ou technique parmi ses coreligionnaires en Orient. Il appelle spécialement mon attention sur ce fait que les écoles fondées et entretenues par l'Alliance ont à leur tête des maîtres élevés à Paris, munis de brevet de capacité délivré à l'Hôtel de Ville et enseignant en français. Trente établissements scolaires, contenant actuellement 4991 élèves des deux sexes, sont déjà ouverts dans les États musulmans du bassin de la Méditerranée. [...]

Il me paraît que l'Alliance israélite se rattache aux intérêts sur lesquels doit s'étendre notre sollicitude en Perse et vous êtes invité à prêter vos bons offices aux écoles qu'elle entretient ainsi qu'aux maîtres qui les desservent. Recevez, Monsieur, les assurances de ma considération la plus distinguée ».

En 1892 le ministre Ribot incita le ministre René de Balloy à accorder son secours aux écoles de l'Alliance Israélite Universelle, sans clairement faire allusion à l'hostilité dont étaient victimes les juifs d'Hamadan au même moment. De Téhéran René de Balloy eut beau jeu de lui répondre qu'aucune école en Perse ne relevait encore de l'AIU. Mais un an plus tard, à la suite d'une lettre du délégué apostolique Mgr Montéty qui dénonçait les sévices subis par les juifs d'Ourmieh, René de Balloy avoua dans une dépêche adressée à Paris : «Je ne puis dissimuler à Votre Excellence qu'il est très difficile aux autorités centrales de réagir contre les préjugés dont toute la population est animée à l'égard des juifs. Le chah, le grand vizir, les ministres n'ordonneront jamais de persécution contre les israélites ; mais ils sont à peu près impuissants à les protéger contre les avanies de tous les jours $» 53$.

Les ministres français des Affaires étrangères se montrèrent donc enclins à encourager les écoles de l'AIU dont l'enseignement faisait largement appel à la langue française, au détriment des autres écoles.

\section{L'adoption du bahaïsme par de nombreux israélites de Perse}

Au milieu du XIX ${ }^{\mathrm{e}}$ siècle le Bab avait prêché en Perse faveur d'une réforme de l'islam. Il fut mis à mort à Tabriz en 1850. Son successeur fut d'abord Mirza Yahya 'Azal qui s'inclina finalement devant Hosseyn 'Ali Nouri Baha'ullah. Certains demeurèrent disciples du Bab, les autres suivirent Baha'ullah qui s'installa à Akka/Acre en Palestine ottomane. Prêchant la

\footnotetext{
${ }^{52}$ AFMAE, C. P., Perse, vol. 38, Paris 4 avril 1879. Les archives de l'AIU conservent de telles demandes.

${ }^{53}$ AMFAE, C. P., Perse, vol. 44 et 45, Paris 26 septembre 1892 et Téhéran 25 mars 1893.
} 
tolérance et l'abolition des rites et pratiques discriminatoires, le babisme, puis le bahaïsme exercèrent un grand attrait sur bon nombre d'Iraniens, en dépit des persécutions engagées contre eux. Le danger pour les écoles presbytérie ne provint pas donc seulement de l'hostilité des rabbins ou des modjtaheds, mais aussi de l'attirance des israélites vers le babisme ou le bahaïsme. Cette question a fait l'objet de nombreuses études telles celles de W. Fischel en 1934, «The Bahaï movement and Persian Jewry (Jewish Review), de M. Momen en 1984, «A preliminary survey of the Bahaï Community of Iran during the Nineteenth century » (Iran im 19. Jahrhundert und die Entstehung der Bahaï Religion) ou plus récemment la thèse de M. Amanat, soutenue à Los Angeles en 2006 " Negociating Identities : Iranian Jews, Muslims and Bahais in the memoirs of Rayhan Rayhani (1859-1939) ${ }^{54}$. Les presbyteriens ressentirent très vite que le bahaïsme qui attirait les familles israélites de Perse risquait de les concurrencer.

Les diplomates français, de leur côté, insistèrent sur les risques encourus en Perse par les communautés israélites car les juifs qui se faisaient babis ou bahaïs - babis et bahaïs étaient souvent confondus - restaient, semble-t-il, dans le quartier juif et faisaient peser des menaces sur la communauté tout entière dans la mesure où modjtaheds, mollahs ou gouverneurs s'en prenaient aux «rénégats babis », et donc au quartier dans lequel ils résidaient, en pensant défendre la communauté musulmane. C'est ce qu'expliqua à son supérieur à Paris Joseph de Mareuil, chargé d'affaires à Téhéran entre septembre et décembre $1879^{55}$, en détaillant ses interventions et celle du chargé d'affaires anglais en faveur d'accusés soupçonnés d'être babis :

«Il y a eu vers la fin du mois dernier quelques troubles à Ispahan qui ont également menacé la sécurité des chrétiens de cette ville. Trois frères musulmans, riches négociants qui avaient le tort d'être créanciers d'Imam Djouma pour une somme considérable ont été accusés de babysme et deux d'entre eux ont été mis à mort avec la connivence du prince Zell es-Soltan, fils du Shah et gouverneur de la ville, qui a partagé leurs dépouilles avec l'autorité religieuse. Cette accusation de babysme sert généralement de prétexte pour des cruautés de ce genre et il m'a été rapporté d'ailleurs que les deux accusés avaient renié le Bab, ce qui d'après la loi persane aurait dû suffire pour les faire absoudre. Mais cette manière de procéder a pour résultat de soulever le fanatisme de la population musulmane et, à la faveur du trouble populaire, les exactions peuvent passer inaperçues. Dans cette occasion les missionnaires catholiques et protestants ayant écrit au Prince une lettre en faveur des deux malheureux dont la mort était résolue, se sont trouvés en butte aux menaces des principaux mushteids (sic) qui ne parlaient de rien moins que de soulever la population et de la lancer sur le quartier chrétien. Le supérieur de la mission catholique m'ayant immédiatement télégraphié et l'agent anglais ayant en même temps avisé Monsieur le chargé d'affaires d'Angleterre, nous avons fait une démarche simultanée et urgente à la suite de laquelle des ordres sévères ont été envoyés au gouverneur d'Ispahan. L'ordre s'est trouvé immédiatement rétabli et le troisième accusé a même eu la vie sauve ».

La question du babisme et celle du bahaïsme semblèrent si récurrentes à Téhéran que le chargé d'affaires français Fernand Souhart (chargé d'affaires à Téhéran entre octobre 1882 et février 1883) tenta d'en présenter une synthèse à son ministre à Paris, en essayant de rapprocher christianisme et bahaïsme ${ }^{56}$ :

« Ainsi que Votre Excellence le sait la Perse fut de 1847 à 1852 le théâtre d'un soulèvement religieux qui, commencé d'une manière pacifique, fut étouffé dans le sang. Son chef qui se faisait appelé Bab fut mis à mort et ses disciples périrent dans les tourments les plus raffinés, rappelant en quelque sorte ceux auxquels furent soumis les premiers chrétiens.

\footnotetext{
${ }^{54}$ Ces ouvrages sont référencés dans la bibliographie.

${ }^{55}$ AMFAE, C. P., Perse, vol. 38, Téhéran 20 avril 1879.

${ }^{56}$ AMFAE, C. P., Perse, vol. 39, Téhéran 17 février 1883, « Le babysme en Perse ».
} 
Néanmoins, depuis cette époque, le babysme, loin de s'éteindre, a poussé de profondes racines dans toute la Perse, à tel point qu'aujourd'hui, quand deux Persans se rencontrent, on peut certainement dire que l'un des deux appartient à la secte. Le chah, à l'instigation de ses courtisans ne veut voir dans les Babys que des membres d'une société secrète ayant pour but le renversement du trône de la dynastie des Qadjars. Pour lui ce sont comme les Nihilistes de la Perse. Aussi les ordres les plus sévères sont-ils donnés à l'égard de ces malheureux qui sont obligés de ne pratiquer leur foi que le plus secrètement possible et d'accomplir ouvertement tous les préceptes de l'islam. La semaine dernière une vingtaine d'entre eux ont été dénoncés et, sur l'ordre de Sa Majesté, jetés dans les prisons. [...] Autant qu'on peut en juger, soit par les écrits laissés par le Bab et commentés plus tard par ses disciples, soit par quelques paroles échappées à des Persans qu'on peut soupçonner d'appartenance à la secte nouvelle, les Babys sont des réformateurs, en quelque sorte les protestants chiites de l'islamisme. D'après eux on aurait altéré le Coran. [...] Dans l'ordre économique les Babys demandent la réforme des impôts, prêchent la charité envers les pauvres et veulent qu'on développe le commerce par tous les moyens ; aussi trouvent-ils que le gouvernement actuel n'entre pas en rapport assez direct avec les Européens desquels il y a beaucoup de bonnes choses à apprendre, de là leur haine contre les mollahs qui sont la classe de la population persane la plus hostile aux idées modernes. En résumé ces novateurs sont des gens très doux, très tranquilles se rapprochant beaucoup plus du christianisme que de l'islamisme et ne voulant faire prévaloir leurs idées que par la persuasion ».

Le jugement de Fernand Souhart est intéressant en ce qu'il se faisait l'écho de l'opinion de certains diplomates et intellectuels iraniens de l'époque qui rendaient les mollahs responsables des blocages de l'économie et de la société iraniennes; il montrait aussi combien les occidentaux se sentaient plus proches des Iraniens babis que des Iraniens musulmans fondamentalistes. Il ne dissimulait pas les raisons du rejet des Babis par les musulmans. Si les lettres de 1881 du vazir Mostawfi el-Mamalak avaient autorisé les juifs d'Hamadan à passer d'une religion à l'autre, c'était à condition de ne pas affecter la situation de la communauté musulmane.

Peu après avoir pris en charge la station d'Hamadan en 1882, les presbytériens manifestèrent leur étonnement devant le grand nombre de juifs passés à l'islam. Ils pensèrent que l'islam leur offrait peut-être plus de sécurité que le judaïsme, comme le pensa l'historien H. Lévy en suggèrant que les israélites avaient abandonné la foi de leur père en raison de l'oppression dont ils étaient victimes et de leur pauvreté ${ }^{57}$. Les presbytériens furent ensuite étonnés de rencontrer dans les quartiers juifs d'Hamadan, ou dans les villes environnantes de moindre importance, des Babis ou des Bahaïs qui semblaient vivre avec une assez grande liberté d'esprit. Ils furent par ailleurs déconcertés de constater, d'année en année, que l'émigration de juifs vers Jérusalem ne tarrissait pas, et que persistait le mouvement des juifs vers le bahaïsme, qui pouvait les ammener à rejoindre Akka qui n'était finalement pas éloigné de Jérusalem. Le couple J. W. Hawkes fit halte en avril 1887 à Dowlatabad, non loin d'Hamadan ; l'un et l'autre décrivirent les « babis » qu'ils y rencontrèrent, qui s'inspiraient du Nouveau Testament, vivaient dans l'Age d'Or et rejetaient toute idée de résurrection ${ }^{58}$. La présence de ces Babis fut confirmée par le représentant de l'AIU en 1903 dans une lettre écrite d'Hamadan ${ }^{59}$ : «Des mouvements contre les babis à Ispahan et Yezd ont fait deux cents victimes d'une mort cruelle.[...] A Dowlatabad la foule est venue contre les juifs babis d'Hamadan qui étaient venus y exercer leur commerce. [...] Depuis un mois à Hamadan le gouverneur Salar es-Saltaneh a voulu gouverner sans tenir compte du clergé. Les prêtres ont décidé de fermer les mosquées et d'ameuter contre les babis ».

En 1888, les presbytériens soulignèrent que leur travail auprès des juifs d'Hamadan se trouvait compliqué par le manque d'unité au sein de la communauté juive ; les uns

\footnotetext{
${ }^{57}$ LEVY, H., Tarikh-e Yahoud-e Iran, 3, p. 657.

${ }^{58}$ AP, Hamadan june $16^{\text {th }} 1887$.

${ }^{59}$ AIU, I C2, Hamadan 28 août 1903, « Sur le babisme ».
} 
demeuraient fermement attachés à leurs traditions et à la «vieille loi de Moïse »; ceux-là étaient peu nombreux, leur sembla-t-il ; les autres devenaient «babis, musulmans, mais, hélas! ils n'étaient pas devenus chrétiens » ${ }^{60}$. Ils apprécièrent au cours de l'été 1890 les visites spontanées de femmes juives, qui se déclaraient «babies », venues avec leur samovar pour lire le Nouveau Testament autour de verres de thé. Le missionnaire Holmes établit en 1896 une relation serrée entre christianisme et bahaïsme : "The Jews here as in Hamadan and many other parts of Persia are largely with the doctrine of Beha, the successor of the Bab and as these accept Christianity as a preparation element in the evolution of Behaism it is easy for them to profess faith in Christ, these profession to a cross examination before accepting them as genuine ». Au cours le l'année 1896-1897, les classes de la Jewish Girls' school presbytérienne se tinrent d'ailleurs dans la maison d'un Bahaï.

Pourtant en 1895 Joseph Somekh était venu de Bagdad étudier les possibilités d'implantation d'écoles de l'AIU à Hamadan. Son rapport fut plutôt pessimiste ${ }^{61}$ :

« [...] Un autre malheur qui les [les Israélites d'Hamadan] accable et les avilit de jour en jour, c'est l'inconstance de leur religion. Environ cinq cents Israélites ont embrassé le mahométisme et le christianisme. Les uns pour se soustraire aux persécutions musulmanes, les autres par propre plaisir. Plusieurs d'entre eux professent la nouvelle religion des Babis. Ils reconnaissent une doctrine établie par un prophète qui vivait, disent-ils, à Akka.

Résultat de tout cela : c'est que le judaïsme de Hamadan et de Perse en général restera ce qu'il est et il n'y a pas moyen de le relever de son assoupissement jusqu'à ce que la face et les mœurs de la Perse prendront une autre forme. La création d'une école en Perse est impossible par l'Alliance car elle rencontre des difficultés tout à fait insurmontables ».

\section{Conclusion}

En 1898 l'AIU ouvrit une école à Téhéran ; Joseph Cazès son directeur demanda à ses trois cents élèves de porter un uniforme sur lequel le port d'une étoffe rouge (réservé aux israélites) fut remplacé par les deux mains jointes, emblème de l'AIU. Ils se rendirent ainsi chez le vazir. En 1900 des écoles de 1'AIU furent ouvertes à Hamadan et Ispahan, en 1901, à Kachan, en 1903, à Senneh, Chiraz, puis Kermanchah. En 1908 les onze écoles de l'AIU accueillaient 2225 élèves. On serait tenté de lire en filigrane, derrière ce bilan, l'échec des écoles presbytériennes destinées aux enfants juifs de Perse, ouvertes pendant deux ou trois décennies et dans deux villes seulement - Téhéran et Hamadan, si l'on exclut Ourmieh où l'enseignement des presbytériens aux enfants juifs fut très diffus avant 1900. Il faut dépasser les chiffres et saluer les initiatives des presbytériens qui ont répondu à une demande avérée d'enseignement de la part de familles israélites déçues par l'enseignement dispensé dans les écoles des synagogues, enseignement qui ne s'adressait que très rarement aux filles. Les presbytériens ont permis de secouer le monopole jalousement conservé par les rabbins. Ils ont proposé un enseignement plus moderne dans leurs primary schools, ont mêlé dans leurs collèges (high schools) des enfants de religions différentes, ont renforcé l'apprentissage de l'hébreu et ont entrepris d'enseigner les petites filles. Ils ont imposé une lecture quotidienne de la Bible qui semble avoir répondu à une quête spirituelle, qui était aussi celle des juifs devenus bahaïs qui n'ont pas hésité pas à se joindre à eux pour lire le Nouveau Testament. Mais en proposant aux enfants juifs scolarisés dans leurs écoles de devenir chrétiens, ils ont risqué de les soustraire à leur communauté. Quand en 1898 Joseph Cazès a ouvert les portes de la première école de l'Alliance Israélite Universelle en Perse, les élèves juifs des écoles presbytériennes ont exprimé leur crainte de se couper définitivement de leur communauté s'ils ne rejoignaient pas les écoles de l'AIU. Or en Perse il était difficile à un individu de vivre

\footnotetext{
${ }^{60}$ AP, Hamadan, Jewish Work, 1888.

${ }^{61}$ AIU, AI B11, Hamadan 15 mars 1895. Les termes sont soulignés dans le texte.
} 
hors de sa communauté. Il se retrouvait sans protection. Les envoyés de l'AIU n'ont pas estimé que les écoles presbytériennes leur avaient fait de l'ombre ou que leurs high schools risquaient de leur en faire; le danger, pour eux, a été l'attrait des bahaïs; ils ont donc inauguré un enseignement non confessionnel, à l'écart des «chiites fanatiques et des babistes exaltés », si l'on en croit M. Bassan à Hamadan ${ }^{62}$, enseignement susceptible de renforcer l'unité de la communauté israélite.

\section{Bibliographie}

Archives du ministère français des Affaires étrangères : AMFAE :

Série Correspondance Politique C. P., sous-série Perse, vol. 38, 39, 40, 41, 42, 43, 44.

Série Affaires Diverses Politiques A. D. P., sous-série Perse, vol. 66, 90.

Archives de la Société d'histoire des Presbytériens : AP.

Archives de l'Alliance Israélite Universelle : AIU.

A.I. U. Bulletin annuel, 66, 1904.

A.I.U. Bulletin mensuel, 31, 1903, p. 104 ; 66, 1904, p. 169.

A. I. U. France, I D. I, Statistiques 1903-1904.

A.I.U. France, V. II, D.36, 1879.

A. I. U. France VII D. 36 « Ambassade de Perse » 18 juillet 1873 ; « Tauris ».

A. I. U. France I C.4 Nice 29 septembre 1881.

A.I.U. Iran, AI B11; AI C2.

AdLeR, E., Jews in many lands, Philadelphia, Jewish Publication Society of America, 1905.

AFARY, J., «From outcastes to citizens: Jews in Qajar Iran », Sarshar, Esther's children, Beverly Hills, California Center for Iranian Jewish Oral History, 2002.

Algar, H., Religion and State in Iran, 1785-1906, Berkeley Los Angeles: University of California Press, 1969.

Amanat, A., Pivot of the Universe, Berkeley and Los Angeles, University of California Press, 1977.

ANET, C., «Les écoles israélites en Perse », L’Univers israélite, 61, 1905, p. 468.

Aubin, E., La Perse d'aujourd'hui, Paris, 1908.

BAssetT, J., Persia, the Land of the Imams, London, Blackie \& Son, 1887.

BENJAMIN II (Israel ben Yousef), J. J., Eight years in Asia and Africa, from 1846 to 1855, Hanover, 1859.

BIRD (Bishop), I., Journeys in Persia and Kurdistan, London, John Murray, 1891.

BRUCE, J. and GARLAND, J.L., The Jews of Persia: their past history and present condition, London: London Society for Promoting Christianity amongst the Jews, 1909.

Chaoulli, A., Les musiciens juifs aux XIX et XX $X^{e}$ siècles, Paris, L'Harmattan, 2006.

CONFINO, A., "La communauté juive d'Ispahan », Revue des Ecoles de l'Alliance Israélite Universelle, 3, octobre-décembre 1901, p. 186.

Confino, A., L'action de l'Alliance Israélite en Perse, n. d. (1947 ?), Alger, Imprimerie Charros.

ConfINo, A., «Establishment of the Alliance School in Tehran », Padyavand Judeo-Iranian and Jewish Studies Series, vol. 3, p. 93-108 et p. 261-284 (en persan).

Curzon, G., Persia and the Persia question, London: Longmans, Green \& Co, 1892.

\footnotetext{
${ }^{62}$ AIU, I C2, Hamadan 20 février 1902, n 47.
} 
FISCHEL, W. J., «The Bahai movement and Persian Jewry », The Jewish Review, London, Socino 1934, p. 53-54.

FISCHEL, W. J., «The Jews of Persia », Jewish Social Studies, vol. 12, avril 1950.

FISCHEL, W. J , «Azarbaijan in Jewish history », Proccedings of the American Academy for Jewish research vol. XXII; 1953.

FISCHEL, W. J., «The Jews of Kurdistan a hundred years ago », Conference on Jewish relations

New York 1944, reprinted from Jewish Social Studies vol. VI, 3.

GARLAND, J. L., The Hebrew Christian as a missionary power, London: London Society for Promoting Christianity among the Jews n.d.

GoBINEAU A. de, Les religions et les philosophies dans l'Asie Centrale, Paris, Les Editions G. Crès, 1923.

HAKIMIAN, H., « Wage labor and migration:Persia workers in southern Russia, 1880-1914 », IJMES, 17, 1985, p. 443-462.

HounTUN-SCHINDLER, A., Eastern Persian Irak,London, 1896.

LEVEn, N., Cinquante ans d'histoire L'Alliance israélite universelle (1860-1910), Paris Librairie Félix Alcan, 1911.

LEVY, H., Tarikh-e Yahudi-ye Iran, Tehran, Kitab Forouch-e Biroukhim, 1960.

MARTIN, V., The Qajar Pact, London and New York :I.B. Tauris, 2005.

MOMEN, M., The Babi and Bahai religions 1844-1944, Oxford, 1981.

Momen, M., «A preliminary Survey of the Bahai-Community of Iran during the Nineteenth century », Iran im 19. Jahrhundert und die Entstehung der Bahaii religion, éd. J. C. Burgel and I. Schayani, Hildesheim, Zurich, New York : Goerg Olms Verlag, 1998.

NATEQ, H., «Tarikhchah-ye Allians Israeli dar Iran», Yahudiyan-e Irani dar tarikh-e Mou'asin, éd. H. Sarshar, Beverly Hills, California, Center for Iranian Jewish Oral History, 1997, 2, p. 55-130.

Netzer, A., «The fate of the Jewish community of Tabriz », Studies in Islamic History and Civilization in honour of professor David Alayon, ed. M. Sharon, Leiden: Brill, 1986, p. 411419.

Netzer, A., The Jews of Persia and Alliance in the late $19^{\text {th }}$ century some aspects, The Hebrew University of Jerusalem, 1986.

Netzer, A., «Hanina Mizrahi, a distinguished personality from Tehran », Padyavand, JudeoIranian and Jewish studies, ed. A. Netzer, vol. 3, p. 165-171 et p. 361-371 (en persan).

POLAK, J. E., «Persécution israélite », Archives israélites 26 (1865).

RINGER, M., Education, Religion and the discourse of Cultural Reform in Qajar Iran, Costa Mesa, Mazda, 2001.

SAHIM, H., «Jews of Iran in the Qajar period: Persecution and perseverance », Religion and society in Qajar Iran, éd. R. Gleave, London and New York : Routledge Curzon, 2005, p. 293-310.

SANDER, D., «Juifs et Bahaïs en Iran, 1844-1920», Revue des Études juives, 163, 1-2, janvier-juin 2004, p. 257-271.

SPEER, R. E., Report on the Perisa Missions of the Presbyterian Board of Foreign Missions, The Board of Foreign Missions of the Presbyterian Church in the USA, New York, 1897.

STERn, H.A., Downings of light in the East, London, Charles H. Purday, 1854.

TSADIK, D., «The legal status of religious minorities: Imami Shi'i law and Iran's Constitutional Revolution », ILS, 10, (2003) p. 379-405.

TSADIK, D., «Religious disputations of Imami Shi'is against Judaism in the late eighteenth and nineteenth centuries », Studia Iranica, 34 (2005) p. 95-135.

Waterfield, R. E., Christians in Persia, London, George Allen \& Unwin, 1973. 
WOLFF, J., Researches and missionary labours among the Jews, Mohammedans and other sects, London, 1835. 